\title{
Global 30-240 keV proton precipitation in the 17-18 April 2002 geomagnetic storms:
}

\section{Conductances and beam spreading}

\author{
Xiaohua Fang, ${ }^{1}$ Michael W. Liemohn, ${ }^{1}$ Janet U. Kozyra, ${ }^{1}$ and David S. Evans ${ }^{2}$ \\ Received 9 October 2006; revised 22 January 2007; accepted 1 February 2007; published 9 May 2007.
}

[1] We investigate the global Pedersen and Hall conductance distribution during the

17-18 April 2002 geomagnetic storms by combining NOAA/POES 30-240 keV proton precipitation measurements with three-dimensional (3-D) particle transport modeling. Significant conductances are generated due to energetic proton precipitation in the selected storms, with peak values up to $\sim 10 \mathrm{~S}$ in the dusk sector. The effect of horizontal beam spread on the conductances, which is a unique feature of ion precipitation, is first assessed in this paper. It is found that with the neglect of the beam spread, an overestimation (up to 10\%) occurs at the center of the major proton precipitation region. At the edge (particularly at the equatorward edge), a severe underestimation (around $-50 \%$ or worse) is induced. Moreover, 3-D scattering extends the equatorward edge of the high conductance region down to around $55^{\circ}$ magnetic latitude in the storms. A significance domain (up to $4.4^{\circ}$ ) exists equatorward of the peak proton precipitation region, in which significant conductances would be seriously underestimated without the beam spreading effect. It is also found that the beam spreading effect is more significant for the Pedersen conductances than for the Hall conductances because of the altitude dependence of both the conductivity profiles and the spreading. The findings emphasize the role of proton precipitation as well as its associated beam spreading, especially at the equatorward edge of the precipitation zone in the evening sector, where proton precipitation-produced conductivity can influence storm-time phenomena, such as subauroral polarization streams, ionospheric storm enhanced density structures, and inner magnetospheric plasma dynamics (i.e., the ring current and plasmasphere).

Citation: Fang, X., M. W. Liemohn, J. U. Kozyra, and D. S. Evans (2007), Global 30-240 keV proton precipitation in the 17-18 April 2002 geomagnetic storms: 2. Conductances and beam spreading, J. Geophys. Res., 112, A05302, doi:10.1029/2006JA012113.

\section{Introduction}

[2] Precipitating particles from the magnetosphere are a major energy source impacting the upper atmosphere at high latitudes, dominantly on the nightside. In general, most of the particle energy input comes from electrons with protons contributing only around 15\% [Hardy et al., 1989; Galand et al., 2001]. However, at times and persistently in some regions (i.e., the duskside auroral oval and the cusp), precipitating ions can be a significant or dominant energy source for the ionosphere-thermosphere system [Sharber, 1981; Gussenhoven et al., 1987; Hardy et al., 1989; Galand et al., 2001]. In fact, high-energy protons are more efficient at ionizing neutrals through ionization and charge exchange collisions than electrons [Strickland et al., 1993; Galand et al., 1999]. The ionization enhancement by

\footnotetext{
${ }^{1}$ Space Physics Research Laboratory, University of Michigan, Ann Arbor, Michigan, USA.

${ }^{2}$ Space Environment Center, NOAA, Boulder, Colorado, USA.

Copyright 2007 by the American Geophysical Union. 0148-0227/07/2006JA012113
}

particle precipitation has a direct impact on the ionospheric electron density. The comparisons of satellite observed proton precipitation together with ground based radar measurements have revealed that in some regions proton input alone can account for the altitude profiles of ionospheric electrons [Basu et al., 1987; Senior et al., 1987].

[3] Another important impact of particle precipitation is on electrical conductivities by augmenting the ionospheric electron concentration. The understanding of high-latitude ionospheric electrodynamics patterns and current systems relies on an accurate modeling of the ionospheric conductance, that is, height-integrated conductivities [e.g., Richmond and Kamide, 1988]. The ionospheric conductance can also regulate magnetosphere-ionosphere energy exchange through coupled electrodynamic processes [Fedder and Lyon, 1987; Raeder et al., 2001; Khazanov et al., 2003; Ridley et al., 2004; Liemohn et al., 2005]. In addition, Joule heating is directly dependent on the Pedersen conductivity. Lu et al. [2001] reported that a misrepresented conductance distribution could cause an error in the estimate of the hemispheric integrated Joule heating rate by nearly a factor of 2 . 
[4] In the literature, many efforts have been made to construct the global conductance distribution [Wallis and Budzinski, 1981; Spiro et al., 1982; Hardy et al., 1987; Fuller-Rowell and Evans, 1987; Lummerzheim et al., 1991; Brekke and Moen, 1993; Aksnes et al., 2002]. However, precipitating electrons are the only ionization source considered in the aforementioned models. There is a little exception in the Fuller-Rowell and Evans [1987] model, in which proton energy fluxes were included but treated as if they were carried by electrons. This lack of inclusion of proton precipitation is a common limitation in a conductance model for a long time, as the overall proton energy input contributes around $15 \%$ and is thus often overlooked.

[5] Recently, based on statistical auroral electron patterns [Hardy et al., 1985, 1987] and statistical proton patterns [Hardy et al., 1989, 1991], Galand et al. [2001] estimated the enhancement in Pedersen and Hall conductances when proton precipitation was taken into account. Their study highlighted the significance of the proton contribution to conductances due to the offset of the proton oval toward dusk [Hardy et al., 1989]. The calculation of proton ionization rates and thus conductances in their study was performed using a one-dimensional (1-D) multistream model [Galand et al., 1997, 1999; Galand and Richmond, 2001].

[6] Instead of relying on statistical particle precipitation patterns, Coumans et al. [2004] used high time resolution observations of precipitating particles, which were derived from the far ultraviolet (FUV) instruments onboard the Imager for Magnetopause-to-Aurora Global Exploration (IMAGE) satellite, to study and underline the proton contribution to conductances. However, because of the lack of energy spectral information on IMAGE, the mean energies of incident electrons and protons in their study had to resort to empirical models [Hardy et al., 1985, 1991]. As a consequence, uncertainties were generated in the conductance calculation due to the estimation of the mean energies and thus the energy fluxes of precipitating particles.

[7] The studies of Galand et al. [2001] and Coumans et al. [2004] emphasize and raise the interest of the role of precipitating protons in the global conductance distribution. However, a common limitation of both investigations is the lack of inclusion of lateral spreading in the incident ion energy deposition calculation. The beam spreading effect is a unique feature of ion precipitation resulting from the coupling between ion and neutral transport. The charge exchange and electron stripping collisions within a precipitating ion beam cause spreading of the beam across magnetic field lines, broadening the region of ionization and excitation it produces [Davidson, 1965; Johnstone, 1972; Igelesias and Vondrak, 1974; Kozelov, 1993; Synnes et al., 1998; Lorentzen, 2000; Fang et al., 2004, 2005].

[8] A comprehensive study on the beam spreading was recently conducted by Fang et al. [2005] using a threedimensional (3-D) Monte Carlo ion transport model [Fang et al., 2004] for incident proton arcs with a variety of parameters. Their findings illustrate that a correction factor that is introduced for a 1-D model [Jasperse and Basu, 1982] is not enough to account for the beam spreading effect when an incident proton arc has finite dimensions. Even after the application of a correction factor, a significant error in the $1-\mathrm{D}$ results in ionization rates $(\sim 20 \%$ underestimation) still exists at ionization peak altitudes at the center of a proton arc of infinite extent in the longitudinal direction and of $120 \mathrm{~km}$ semiwidth in the latitudinal direction. Furthermore, significant ionization processes occur outside of the precipitation domain due to beam spreading, which cannot be deduced in a 1-D model. However, there is so far a lack of knowledge about to what extent 3-D ion scattering has an effect on the conductance distribution (and thus the magnetosphere-ionosphere coupling) produced by proton precipitation on a global scale. This issue is the main focus of the present study.

[9] In the companion paper [Fang et al., 2007, hereinafter referred to as paper 1], global 30-240 keV proton precipitation patterns are obtained during the 17-18 April 2002 storms using newly developed 3-hour National Oceanic and Atmospheric Administration (NOAA) data products. It is the first attempt of constructing global ion patterns using in situ proton precipitation measurements at a reasonable cadence ( 3 hours). The high correlation between the proton precipitation pattern change and the variation of other geophysical indices well demonstrates that energetic proton precipitation patterns are a valuable tool for investigating the inner magnetospheric activity. In this paper, we will apply the proton precipitation patterns obtained in paper 1 (with the focus on the energy range of 30-240 $\mathrm{keV}$ ) to assess the resulting Pedersen and Hall conductances and evaluate the influence of beam spreading.

[10] An overview of this paper follows. In section 2 the global 30-240 keV proton precipitation patterns constructed in the companion paper (paper 1) are used as a topside energy input at $850 \mathrm{~km}$ altitude during the April 2002 storms. In section 3 a brief description is provided for the 3-D Monte Carlo ion transport model [Fang et al., 2004, 2005], which is the major research tool for the ionization rate calculation. Section 4 shows the global distribution of the resulting ionization rates and corresponding Pedersen and Hall conductivities. The conductivities are further height integrated to give the conductances. The effect of beam spreading is presented for all the results. In section 5 the significance of the beam spreading effect of proton precipitation is discussed. Finally, the paper's findings are summarized in section 6 .

\section{Global $\mathbf{H}^{+}$Precipitation in April 2002}

[11] During 17-18 April 2002, a coronal mass ejection swept pass the Earth. The arrival of a strong solar wind dynamic pressure impulse together with highly fluctuating interplanetary magnetic field preceding the interplanetary coronal mass ejection (ICME) triggered a moderate geomagnetic storm, starting on 17 April 2002 at 1107 universal time (UT). The magnetic cloud itself reached the Earth around 0000 UT on 18 April 2002. The present study focuses on the energetic proton precipitation during these time intervals and attempts to understand the interaction of incident protons with the Earth's upper atmosphere.

[12] In paper 1, newly developed data products were proposed for the construction of the global maps of proton precipitation using multiple passes of the NOAA Polar Orbiting Environmental Satellites (POES). The 16-s averaged NOAA/POES energetic particle data were extracted for a 3-hour time interval. The center times of these 
April 2002, Northern Hemisphere, 850 km Altitude

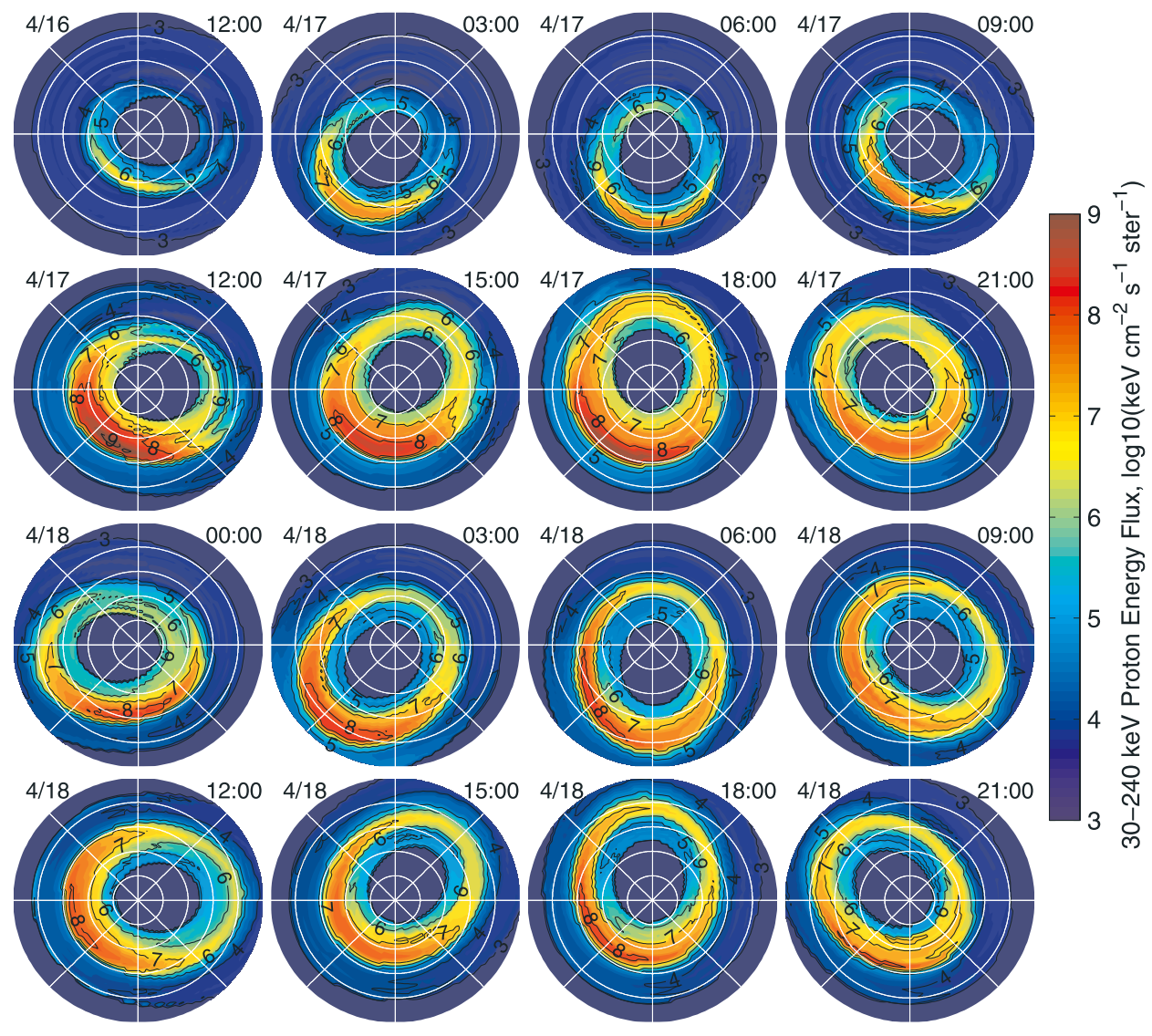

Figure 1. Global patterns of precipitating $30-240 \mathrm{keV}$ proton energy fluxes in the April 2002 magnetic storms. The patterns are displayed in geographic coordinates, with local noon to the top and dawn to the right. The perimeter in geographic latitude is $40^{\circ}$ north and the solid circles are $10^{\circ}$ apart. Time tags indicate the center time of each 3-hour time interval. The first pattern is for 1200 UT on 16 April. The rest shows the patterns at a 3-hour cadence, starting from 0300 UT on 17 April and ending at 2100 UT on 18 April.

intervals for each day are 0000, 0300, 0600, 0900, 1200, 1500,1800 , and 2100 UT. It is presumed that no severe fluctuation in particle precipitation occurred during each 3-hour time window. The validity of the selection of a 3-hour time window has been justified through the close relationship between the generated patterns and other geophysical indices. These generated maps at a 3-hour cadence provide new information on the development and variability in the structure of global $30-240 \mathrm{keV}$ ion precipitation on a timescale commensurate with the growth and decay of the plasma sheet and ring current.

[13] Figure 1 presents the global energy flux distributions of precipitating $30-240 \mathrm{keV}$ protons. These maps are similar to those demonstrated in paper 1 but organized in geographic coordinates. A magnetic field model [Raben et al., 1995] has been applied to convert the patterns in geomagnetic coordinates (as shown in paper 1) to the maps at $850 \mathrm{~km}$ altitude in geographic longitudes and latitudes (as shown in Figure 1). Note that due to the limitation of the NOAA satellite coverage, the patterns are in the corrected magnetic latitude (CML) range of $45^{\circ}-76^{\circ}$ (see paper 1 for more details). The development of global proton precipitation has been quantitatively analyzed in paper 1 in the context of the interaction between the solar wind driving and the inner magnetosphere and therefore is not repeated here. The precipitation maps in Figure 1 provide topside boundary conditions for the ion transport calculation. Ionization and conductivities will be derived and compared with the beam spreading effect neglected or considered.

\section{Model Description}

\subsection{3-D Monte Carlo Model}

[14] The major research tool for the present study is a 3-D Monte Carlo ion transport model, which is described in detail by Fang et al. [2004, 2005]. The Monte Carlo method monitors the trajectories of a large set of precipitating test protons in a collision-by-collision manner, and records the energy degradation in the inelastic (charge exchange, electron stripping, ionization, and excitation) and elastic interactions with ambient neutrals. A full three-species atmosphere $\left(\mathrm{O}, \mathrm{N}_{2}, \mathrm{O}_{2}\right)$ is specified using the Mass Spectrometer and Incoherent Scatter model (MSIS-90) [Hedin, 1991]. As justified by observations from sounding rockets and satellites [e.g., Søraas et al., 1974; Lundblad et al., 1979; Urban, 1981], an isotropic angular distribution is 


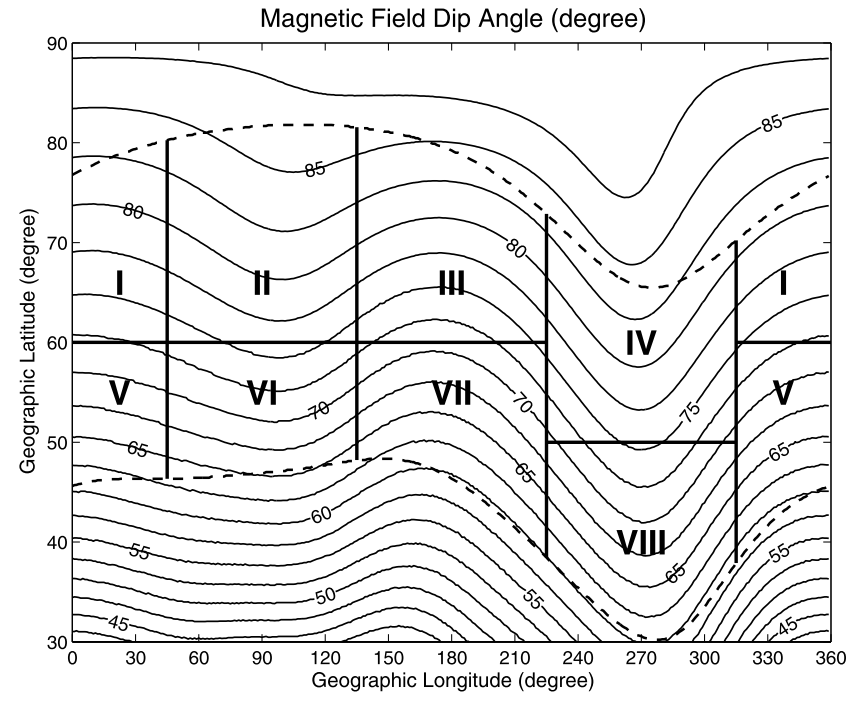

Figure 2. Magnetic field dip angle at $850 \mathrm{~km}$ altitude. The top boundary partition is superposed for 17 April 2002, 1200 UT as an example (see text). The dashed curves indicate the limits for the upper model boundary in magnetic latitude $\left(45^{\circ}\right.$ and $\left.76^{\circ}\right)$.

imposed for the precipitating protons at the upper model boundary $(850 \mathrm{~km}$ altitude). Ionization rates are determined by the ionization and charge exchange impact collisions of precipitating protons as well as generated hot hydrogen atoms. In the ionization rate calculation, the magnetic mirroring effect due to nonparallel magnetic field lines is neglected. The ionization by the secondary electrons, which is not included in the present model, can be roughly estimated by multiplying the primary ionization rate by $0.006 E_{0}$ [Lilensten and Galand, 1998]. $E_{0}$ is the characteristic energy of a Maxwellian proton flux in $\mathrm{keV}$. It should be noted that atmospheric hydrogen is omitted from consideration, given the fact that the collisions of precipitating hot protons with the atmosphere mostly occur at altitudes lower than $500 \mathrm{~km}$ [e.g., Fang et al., 2004], where the atmospheric hydrogen concentration is many orders of magnitude lower than the others.

[15] In our Monte Carlo model, particle transport is described in a natural collision-by-collision algorithm. A large number of test particles are launched in simulations and traced wherever they may go. The lateral spreading of a proton arc, which cannot be handled in a 1-D model, is naturally simulated in the study.

\subsection{Numerical Technique}

[16] A numerical approach has been described by Fang et al. [2004] for calculating the resulting ionization rates for an incident proton arc with finite width. Unlike a 1-D model in which only the vertical (magnetic field aligned) dimension is involved and a spatially infinite topside boundary is assumed with a uniform particle precipitation condition, the horizontal spreading can be and should be considered in a 3-D model with a topside limited area domain. The numerical scheme employed here is basically the same, but the situation is much more complicated. The atmosphere profile and the geomagnetic field vary with location from a global perspective. It is in contrast to the simplified approx- imation made by Fang et al. [2004, 2005], in which regional precipitating protons move in fixed background conditions. Note that in the models of Galand et al. [2001] and Coumans et al. [2004], a simplification was similarly assumed. That is, the ionization calculation yielded in fixed conditions was globally applied to generate the conductance distribution. This limitation is eliminated in our present study.

[17] To allow for the longitudinal and latitudinal dependence of an atmospheric density profile and the magnetic field, and thus for the spatial spreading for an incident proton beam, the topside boundary $\left(45^{\circ}<\mathrm{CML}<76^{\circ}\right)$ is partitioned into 8 parts. There are four $90^{\circ}$ domains in longitude, centered at dayside, dusk, midnight, and dawn, respectively. Each domain is further separated into two parts: high and low latitudes. For an incident proton beam with a given pitch angle distribution and energy, it is assumed that all the location points in each of the 8 boundary partitions correspond to a certain spatial spreading. The spreading effect is calculated by applying our 3-D Monte Carlo ion transport model [Fang et al., 2004] in a planeparallel atmosphere and a uniform (but tilted) magnetic field, whose characteristics are determined at the center of each boundary partition. Figure 2 demonstrates the magnetic field dip angles at $850 \mathrm{~km}$ altitude with the top boundary partition superposed for 17 April 2002, 1200 UT as an example.

[18] For any point of interest (destination point $P$ ) below $850 \mathrm{~km}$ altitude, we applied the International Geomagnetic Reference Field model (IGRF-90) [Langel, 1992] to obtain the magnetic field direction at that point, and then geographically projected in a straight line upward to intersect the top and get a source point $S$. An area centered at $S$, which is $500 \mathrm{~km}$ in longitude and $500 \mathrm{~km}$ in latitude, is regarded as the proton source region to $P$. Our numerical experiments showed that a dimension of $500 \times 500 \mathrm{~km}^{2}$ is enough for a homogeneous energy input to cover $95 \%$ of the ionization rate below $150 \mathrm{~km}$ altitude. The error is dropped to below $\sim 1 \%$ at peak altitudes. Next, the source region is further discretized into a number of $2 \mathrm{~km}$ by $2 \mathrm{~km}$ pixels (source pixel $A$ ). The ionization rate at $P$ therefore can be obtained by accumulating the contributions of proton precipitation from all the source pixels on the $500 \times$ $500 \mathrm{~km}^{2}$ domain.

[19] At each source pixel $A$ at $850 \mathrm{~km}$ altitude, an isotropic proton flux distribution is assumed. The energy input at $A$ is broken up into 31 energy bands to cover the range of 30-240 keV. In each energy band, precipitating protons are assumed to be monoenergetic, with the mean energy of the band and the energy flux calculated using the approximation of a power-law spectrum (see paper 1). As we discussed previously, the characteristics of spatial spreading for the particle transport from $A$ to $P$ are specified depending on which of the 8 boundary partitions the center of the source pixel $A$ is located at. It should be pointed out that a simplified approximation has been made here. Actually we neglect the dependence of the beam spreading (with respect to a magnetic field line) on the change in dip angle within each domain. It has been illustrated by Fang et al. [2005] that even for a $30^{\circ}$ difference, the magnetic field dip angle has a negligible influence except at the edge of a proton arc. As seen in Figure 2, the tilted angle difference 


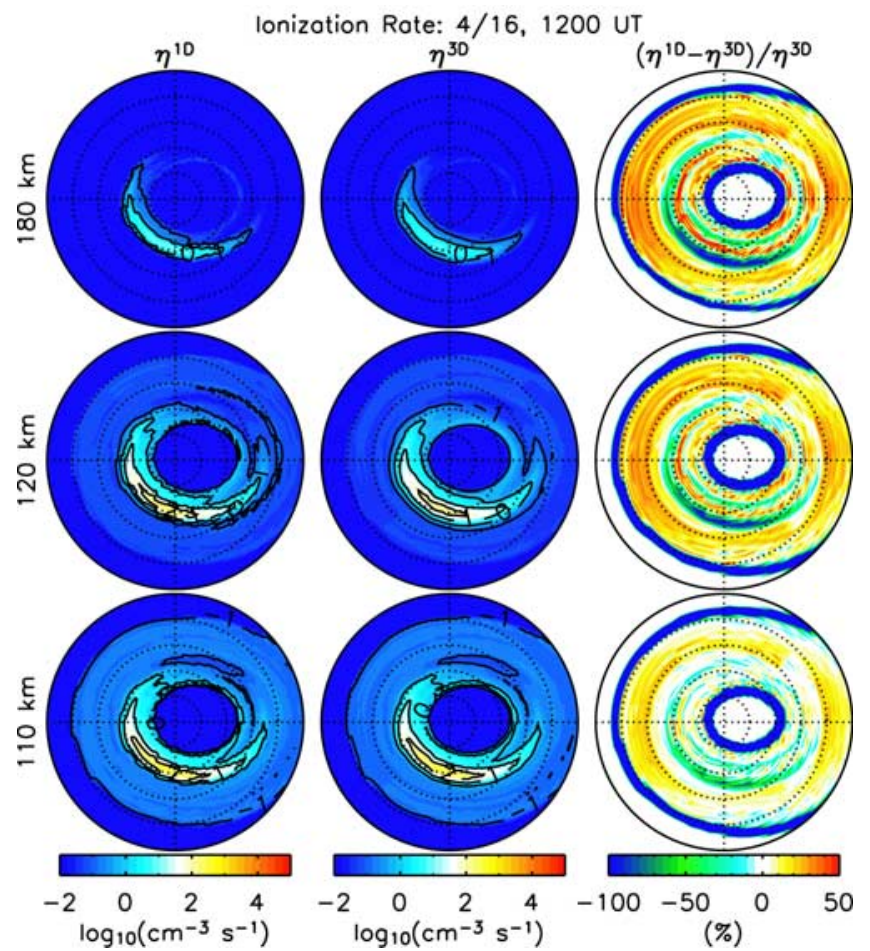

Figure 3. Resulting global ionization rates from 30 to $240 \mathrm{keV}$ proton precipitation centered on 16 April, $1200 \mathrm{UT}$. The results are shown at $180 \mathrm{~km}$ altitude (top row), $120 \mathrm{~km}$ altitude (middle row), and $110 \mathrm{~km}$ altitude (bottom row), respectively. Shown here is ionization rates with the beam spreading effect (left column) neglected, or (middle column) considered, as well as (right column) the relative error of the one-dimensional (1-D) results in percent. The ionization rates are shown on a logarithmic scale with contours every decade. The dial plots have the same format as Figure 1.

between a point in a boundary partition and its center is small (less than $5^{\circ}$ ). Therefore it is safe to assume the same beam spreading with respect to the magnetic field lines for the particles impacting within the same partition domain.

\section{Results}

[20] To achieve the global Pedersen and Hall conductance distribution from 30 to $240 \mathrm{keV}$ proton precipitation during 17-18 April 2002 storms, three steps are performed. First, the resulting ionization rates are calculated using our 3-D Monte Carlo ion transport model [Fang et al., 2004]. Second, electron densities are estimated under the assumption of chemical equilibrium using effective recombination rates. Third, the Pedersen and Hall conductivities are derived and height integrated to yield conductances. The formulations for the last two processes are the same as those described by Galand and Richmond [2001].

\subsection{Ionization Rate}

[21] Figures 3 to 5 present the global ionization rates resulting from the $30-240 \mathrm{keV}$ proton precipitation patterns constructed at three 3-hour time intervals centered at (1) 1200 UT on 16 April, (2) 1200 UT on 17 April, and (3) 0300 UT on 18 April, respectively. These time snapshots are selected to represent three phases during the storm. The first time serves as a quiet reference day, when the solar wind remained very inactive. The second one corresponds to the storm beginning, when high dynamic pressure in the shocked solar wind preceding the interplanetary CME struck the Earth and generated a large amount of proton precipitation to the ionosphere. The third time is associated with a sawtooth event [e.g., Henderson et al., 2006; Clauer et al., 2006], when quasi-periodic particle injections were observed at geosynchronous orbit in a broad range of local time. The ionization rate results are displayed at three altitudes, which correspond to an altitude above the ionization peak $(180 \mathrm{~km})$ and within the ionization peak altitudes in response to 30 $240 \mathrm{keV}$ precipitating protons $(110 \mathrm{~km}$ and $120 \mathrm{~km})$. The beam spreading effect is analyzed at each altitude.

[22] In response to particle precipitation during different storm phases, the ionization rates in Figures 3 to 5 demonstrate distinct characteristics. In the pre-storm condition (16 April, 1200 UT), the ionization resulting from proton precipitation is negligible in magnitude. In the sheath driven storm (17 April, 1200 UT), a pronounced enhancement in ionization is seen in association with the solar wind high dynamic pressure impulse. The main impact occurs in the dusk sector. During the sawtooth event associated with the magnetic cloud driven storm (18 April, 0300 UT), the rise of ionization rates falls into a wider local time range.

[23] Note that the 1-D calculation results shown in this study are obtained by applying our 3-D Monte Carlo model [Fang et al., 2004] but with the lateral diffusion switched off. Rather than relying on a beam spreading correction factor $(\varepsilon<1)$ to adjust the 1-D results as suggested by Jasperse and Basu [1982], we set the correction factor $\varepsilon=1$ to all the 1-D calculations presented in this paper. This is based on the research findings of Fang et al. [2005]. Their comprehensive comparisons between 1-D and 3-D modeling results show that by applying a correction factor to 1-D results, ionization rates would be generally overestimated above $\sim 150 \mathrm{~km}$ altitude and underestimated at lower altitudes (including the ionization peak region). The overestimation is caused by overlooking the difference between the beam spreading for particle fluxes and for ionization rates. The underestimation at low altitudes results from the incorrect assumption made in 1-D models that an incident proton beam has a constant width. Furthermore, the application of a correction factor $\varepsilon<1$ will induce an underestimation in the hemispheric integrated particle energy input. In this sense, a correction factor introduced in the 1-D context is not as useful in practice as expected and therefore is not employed here.

[24] By comparing side by side the ionization results obtained with or without horizontal spatial spreading considered, we can get an overview of how 3-D particle scattering influences the energy deposition patterns. The difference plots in the right columns of Figures 3 to 5 are used to facilitate a comparison of structural details through the relative errors of the 1-D results for each spatial location. The yellow-red shading represents an overestimation (positive error) if the beam spreading effect is ignored, while the green-blue shading represents an underestimation (negative error). Here and throughout the paper, let's focus our attention on the major precipitation zone. For example, as a consequence of the solar wind high dynamic pressure 


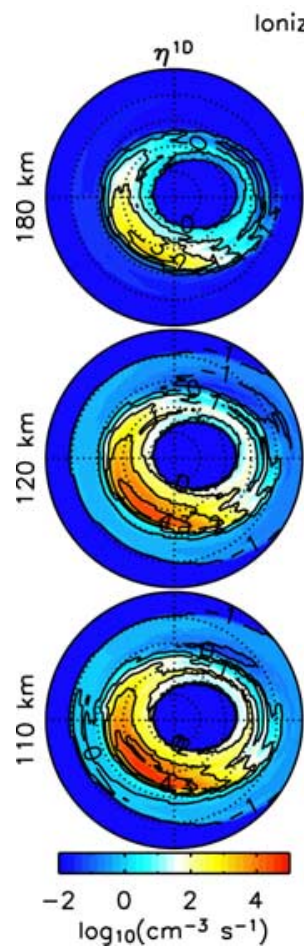

Ionization Rate: 4/17, 1200 UT
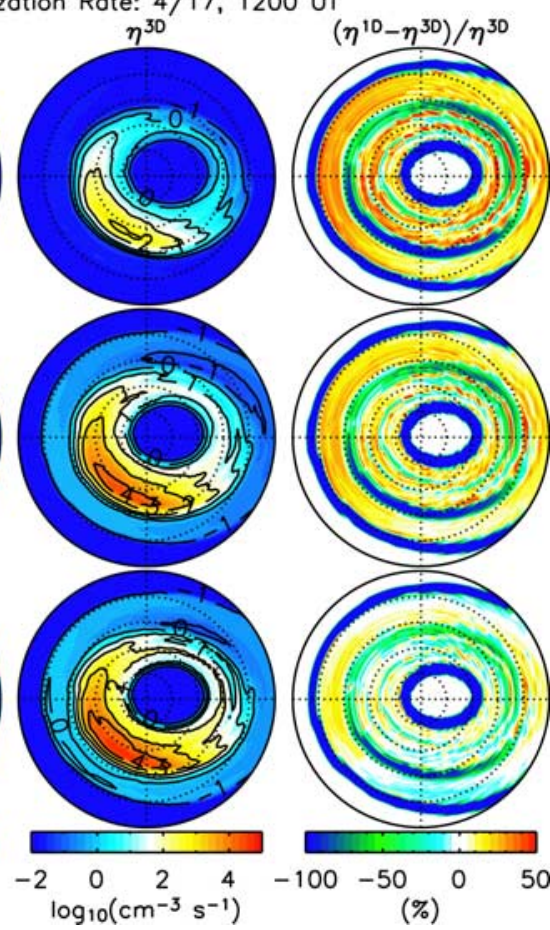

Figure 4. Similar to Figure 3 but for 17 April, 1200 UT.

impinging upon the magnetosphere at 1200 UT on 17 April, a strong duskside precipitation occurred and mostly spanned an approximate latitude range from $65^{\circ}$ to $75^{\circ}$. Far outside of the major precipitation domain, the ionization generated by proton precipitation is more than an order of magnitude lower. It is thus physically less interesting to compare the 1-D/3-D differences there.

[25] As compared in Figures 3 to 5, when the lateral diffusion is considered during proton transport, the atmospheric geoeffectiveness occurs in a broader region, extending to both higher and lower latitudes. This broadening is readily seen along the edges of the major proton precipitation zone as the green-blue regions in the difference plots. For example, in the dusk sector for 1200 UT on 17 April (see the right column of Figure 4), there are green-blue areas at the equatorward edge of the main particle impact domain, spanning a range in latitude from $65^{\circ}$ down to around $60^{\circ}$. A close look at these areas reveals a more smoothly varying boundary in the 3-D results (middle column) than that in the 1-D results (left column). In addition, relatively weaker green regions can be seen along the poleward edge at $\sim 75^{\circ}$. The underestimation of the 1-D results along the edges is also displayed in Figure 3 and Figure 5. It is well illustrated that by considering horizontal spatial spreading in a 3-D model, the physically meaningful domain is extended by several degrees in latitude. It is worth noting that the innermost and outermost blue rings in the 1-D/3-D difference plots also imply the beam spreading effect. However, they occur far from the major precipitation domain and are therefore not discussed further.

[26] Owing to the beam spreading effect associated with proton transport, the small-scale irregularities in the proton precipitation maps in Figure 1, which are easily seen in the longitudinal direction and are introduced in the transformation of the patterns from geomagnetic to geographic coordinates (compare with Figure 8 in paper 1), are washed out and disappear in the 3-D simulation results. On the contrary, the small-scale structures are still retained in the 1-D ionization results. These features are well demonstrated in the direct 1-D and 3-D comparisons for the ionization rates during all the three time snapshots.

[27] There are two interesting common features observed in the detailed comparisons (relative error of the 1-D results) presented in the right columns of Figures 3 to 5. First of all, in the major precipitation zone, the 1-D results have a positive error (yellow and red shading); that is, they are overestimated. On the other hand, at the high- and lowlatitude edges of the major proton impact region, the 1-D results are underestimated (green and blue shading). The overestimation of the 1-D calculation results at the center of a proton oval and underestimation at the edges are understandable, given the extensive discussion of the beam spreading effect by Fang et al. [2005]. To speak briefly, the 1-D calculation implies a simplification to the overhead proton precipitation arc, which is assumed to be infinite with uniform impact. Therefore at the center of a major proton precipitation region, the overestimation of the overhead energy source leads to a higher estimation of the resulting ionization rates. At the edge, the contribution of the nearby high precipitation zone is neglected. As a consequence, an underestimation in ionization rates is inevitable.

[28] It is of interest to note that the beam spreading effect manifests itself more prominently at the equatorward edge than at the poleward edge of the major precipitation zone. This can be explained when viewing the proton precipita-

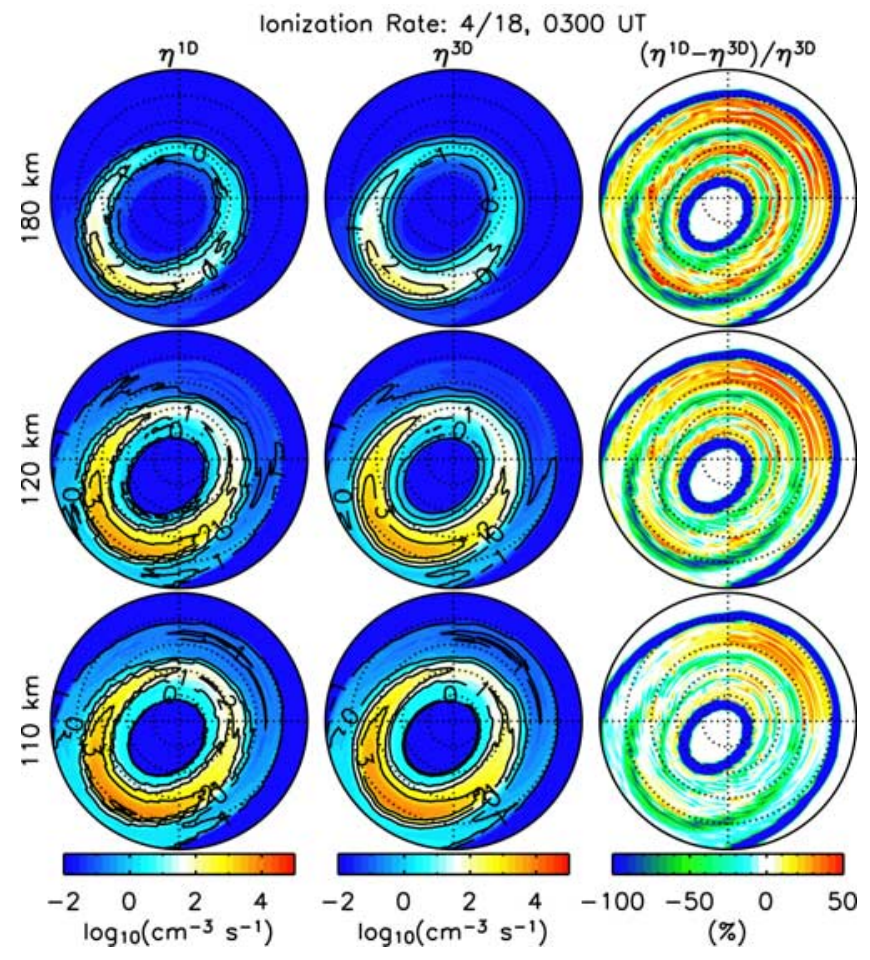

Figure 5. Similar to Figure 3 but for 18 April, 0300 UT. 


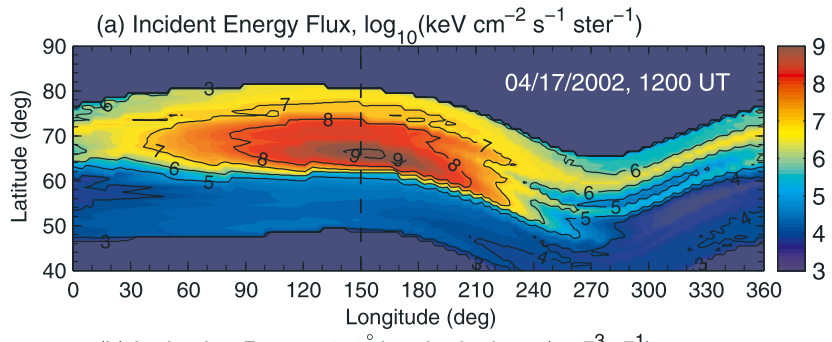

(b) Ionization Rate at $150^{\circ}$ longitude, $\log _{10}\left(\mathrm{~cm}^{-3} \mathrm{~s}^{-1}\right)$

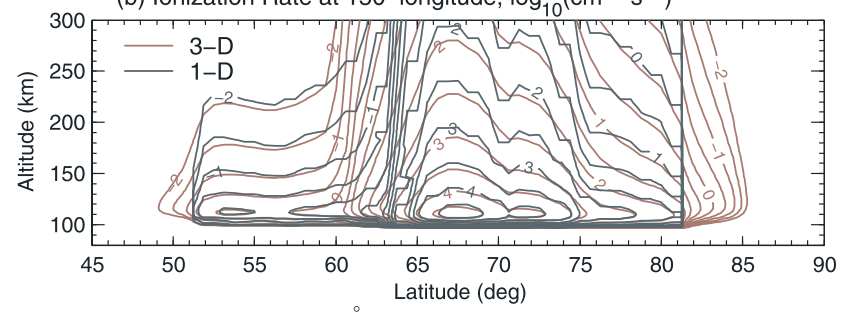

(c) Comparison at $150^{\circ}$ longitude

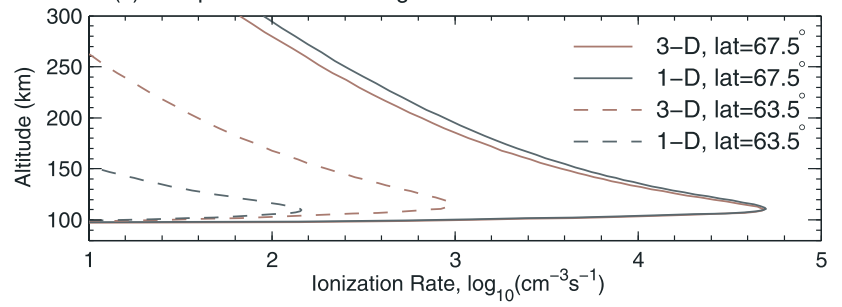

Figure 6. Comparison of the ionization rates on 17 April 2002, 1200 UT, when the spatial spreading effect is considered or neglected. (a) Incident energy flux at $850 \mathrm{~km}$ altitude in geographic longitude-latitude, (b) resulting primary ionization rates at $150^{\circ}$ longitude when the spread effect is considered (red curves) or neglected (black curves), (c) comparison of ionization rates between 3-D and 1-D calculations at $150^{\circ}$ longitude and two latitudes: $67.5^{\circ}$ (solid curves) and $63.5^{\circ}$ (dashed curves).

tion maps in Figure 1. The precipitating particle energy flux has a higher gradient at the equatorward edge, making the error of neglecting the influence of nearby particle energy input larger. Another reason is that the magnetic field lines at higher latitudes are closer to vertical, as seen in Figure 2. It was found by Fang et al. [2005] that more tilted magnetic field lines are associated with a larger (but modest) beam spreading.

[29] Second, the horizontal beam spreading has a stronger effect at higher altitudes. That is, the relative error of the 1-D results (overestimation at the precipitation center and underestimation at the edges) declines with decreasing altitude. This is consistent with the theoretical analysis conducted by Fang et al. [2005]. The key point is that the effective beam radius for an incident proton beam is not invariant with altitude. Rather, it decreases with decreasing altitude below $\sim 300 \mathrm{~km}$ [Kozelov, 1993; Fang et al., 2004]. As a result, the beam spreading effect is weaker at lower altitudes, making the 1-D results closer to the 3-D results.

[30] Let us proceed with the implementation of a quantitative analysis of the influence of spatial spreading on the ionization rates. As an example, Figure 6 provides a detailed comparison between the 1-D and 3-D calculation results on
17 April 1200 UT; that is, when the spatial spreading effect is turned off or on in the numerical simulation, respectively. Figure $6 \mathrm{a}$ shows the incident $30-240 \mathrm{keV}$ proton energy flux observed by NOAA/POES, the same as the first pattern in the second row of Figure 1 but not in a dial plot. Figure $6 \mathrm{~b}$ presents the 1-D/3-D comparison at a latitude-altitude slice through $150^{\circ}$ geographic longitude (local time of 2200). It is shown that when the spreading effect is considered, there is considerable ionization outside of the precipitation region. Through this example, it is seen that at latitudes higher than around $75^{\circ}$ or lower than around $60^{\circ}$, the $1-\mathrm{D} / 3-\mathrm{D}$ difference does not matter much, as the absolute values of the ionization rates drop quickly away from the major precipitation region. Moreover, if the incident proton beam spreads around local magnetic field lines, the small-scale irregular features in the proton precipitation patterns are washed out, and smoother ionization results are generated.

[31] The ionization rates are further compared in detail at two specific locations $\left(67.5^{\circ}\right.$ and $63.5^{\circ}$ geographic latitudes) in Figure 6c. It is clear that in the center of the proton precipitation $\left(150^{\circ}\right.$ longitude, $67.5^{\circ}$ latitude), the 1-D calculation results are acceptable. In this case, an overestimation of $\sim 35 \%$ is observed above $200 \mathrm{~km}$ altitude. At the lower levels, the 1-D results are in fairly good agreement with the 3-D results. The error drops to $\sim 15 \%$ at $120 \mathrm{~km}$ altitude. However, a large underestimation is made by the 1-D calculation at the edge of the major proton precipitation region. In this case $\left(150^{\circ}\right.$ longitude, $63.5^{\circ}$ latitude), there is a severe underestimation in almost the whole altitude region. The error is worse than $-90 \%$ above $120 \mathrm{~km}$ altitude.

\subsection{Electron Density}

[32] As an intermediate step for the conductivity calculation, electron densities are derived from the ionization rates. In the $E$ layer, a chemical equilibrium is a good approximation, making the ion production rate equal to the ion recombination rate. On the basis of the charge neutrality condition, the electron density is written as

$$
n_{e}=\sqrt{\frac{\eta}{\alpha_{\mathrm{eff}}(h)}},
$$

where $\eta$ is the ionization rate generated by precipitating protons, and $\alpha_{\text {eff }}$ is an effective recombination rate as a function of altitude $h$ given by [Vickrey et al., 1982]

$$
\alpha_{\mathrm{eff}}(h)=2.5 \times 10^{-6} \exp \left[\frac{-h(\mathrm{~km})}{51.2}\right]\left(\mathrm{cm}^{3} \mathrm{~s}^{-1}\right) .
$$

The empirically derived $\alpha_{\text {eff }}$ is valid in the altitude range of about $90-180 \mathrm{~km}$. Equation (1) has been successfully applied to explain the electron density profile observed by Chatanika radar using the proton precipitation measurement of NOAA 6 [Basu et al., 1987; Fang et al., 2004].

[33] Figure 7 shows the comparison of electron densities at different altitudes when the 3-D particle transport is considered or not. After the beam spreading effect is taken into account, the geoeffectiveness of proton precipitation takes place across a broader region, as we have seen in Figures 3-5. Note that the square root in equation (1) 


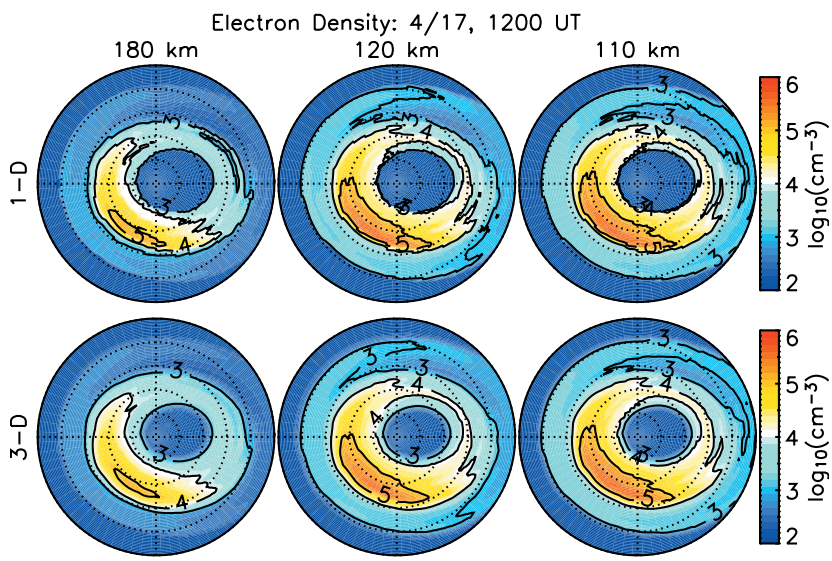

Figure 7. Electron densities resulting from 30 to $240 \mathrm{keV}$ proton precipitation centered on 17 April, 1200 UT. The three columns are the results at $180 \mathrm{~km}, 120 \mathrm{~km}$, and $110 \mathrm{~km}$ altitude, respectively. The beam spreading effect is (top row) neglected or (bottom row) considered in the particle transport calculation. The plots are shown on a logarithmic scale.

makes the relative error of the 1-D electron density results smaller than that of the 1-D ionization rate results.

\subsection{Pedersen and Hall Conductivities}

[34] The Pedersen conductivity $\left(\sigma_{P}\right)$ and Hall conductivity $\left(\sigma_{H}\right)$ can be derived from [e.g., Richmond, 1995]

$$
\begin{gathered}
\sigma_{P}=\sum_{i=\mathrm{O}_{2}^{+}, \mathrm{NO}^{+}, \mathrm{O}^{+}} \frac{n_{i} e}{B}\left(\frac{\nu_{e n \perp} \Omega_{e}}{\nu_{e n \perp}^{2}+\Omega_{e}^{2}}+\frac{\nu_{i n} \Omega_{i}}{\nu_{i n}^{2}+\Omega_{i}^{2}}\right) \\
\sigma_{H}=\sum_{i=\mathrm{O}_{2}^{+}, \mathrm{NO}^{+}, \mathrm{O}^{+}} \frac{n_{i} e}{B}\left(\frac{\Omega_{e}^{2}}{\nu_{e n \perp}^{2}+\Omega_{e}^{2}}-\frac{\Omega_{i}^{2}}{\nu_{i n}^{2}+\Omega_{i}^{2}}\right),
\end{gathered}
$$

where $n_{i}$ is the number density of the ion species labeled by index $i, e$ is elementary charge, $B$ is the strength of the magnetic field, $\Omega_{e}$ and $\Omega_{i}$ are the angular gyrofrequencies for an electron and an ion, respectively. In equations (3) and (4), $\nu_{e n \perp}$ and $\nu_{i n}$ are the electron-neutral (perpendicular to the magnetic field) and ion-neutral collision frequencies. For the $\sigma_{P}$ and $\sigma_{H}$ calculation, we use the same method as described by Galand and Richmond [2001]. That is, the formulas of computing $\nu_{e n \perp}$ and $\nu_{i n}$ are from the survey of Richmond [1995] but with an update on $\nu_{\mathrm{o}^{+}-\mathrm{o}}$ following Pesnell et al. [1993]. As required by the $\nu_{e n \perp}$ and $\nu_{\text {in }}$ computation, the neutral species densities and the neutral temperature are from the MSIS-90 model [Hedin, 1991], while the ion and electron temperatures are from the International Reference Ionosphere model (IRI-90) [Bilitza, 1990]. A branching ratio of an ion species, calculated using the IRI-90 model, is employed to relate $n_{i}$ to the total electron density $n_{e}$. The magnetic field is calculated using the IGRF-90 model [Langel, 1992].

[35] Figure 8 presents the resulting Pedersen and Hall conductivities due to $30-240 \mathrm{keV}$ proton precipitation centered on 17 April, 1200 UT. A notable distinction between $\sigma_{P}$ and $\sigma_{H}$ is that they have different peak locations. The Pedersen conductivities have their maximum values at around $120 \mathrm{~km}$ altitude, while the Hall conductivities peak lower (at around $110 \mathrm{~km}$ altitude). Another interesting feature is that there are considerable Pedersen conductivities at high altitudes (although the values are more than one order of magnitude lower than those at the peak location), while the Hall conductivities at $180 \mathrm{~km}$ altitude are almost negligible.

[36] In Figure 8 the 1-D and 3-D conductivity results are displayed and compared to underline the significance of including 3-D particle transport in a global modeling. To facilitate comparison, 1-D/3-D differences are presented at the bottom of the figure by providing the relative errors of the 1-D results. The same color scale is used as for Figures $3-$ 5. That is, yellow-red shading and green-blue shading represent, respectively, an overestimation of the 1-D conductivity results within the major particle impact domain and an underestimation along the edges. As we discussed in

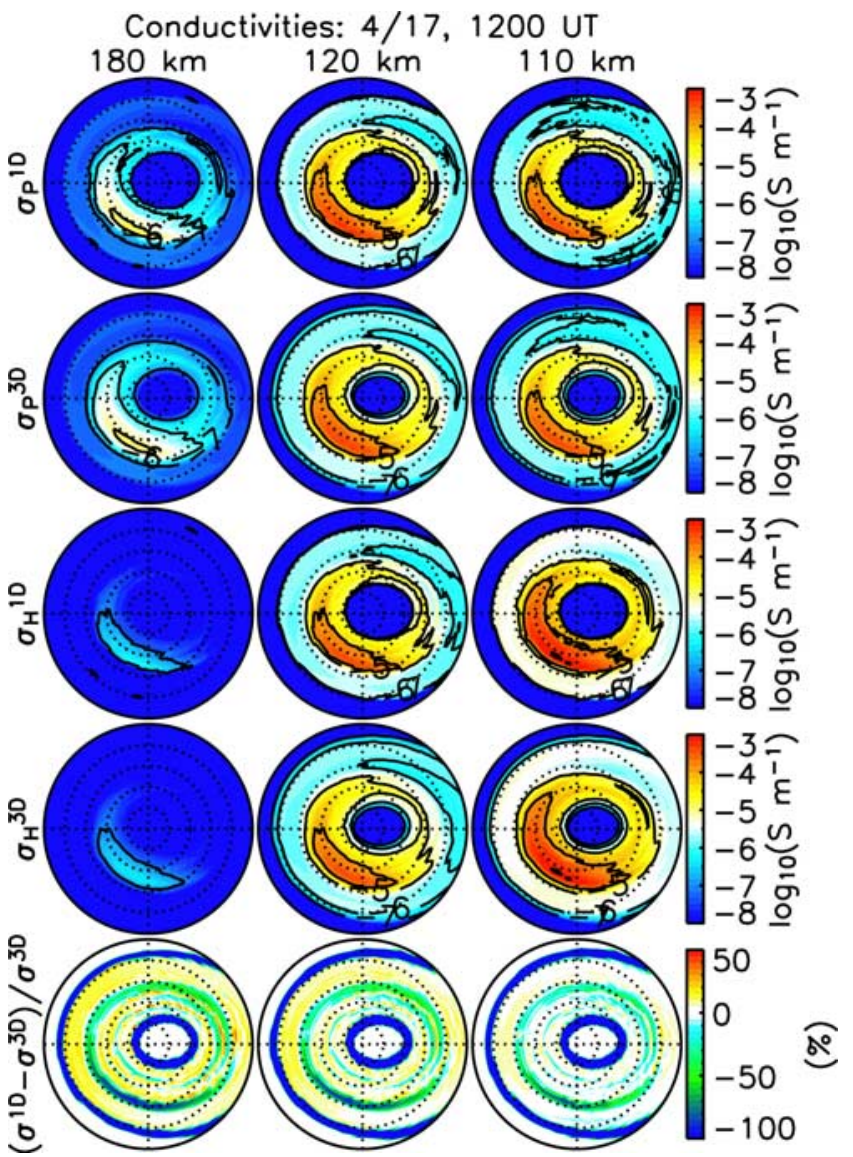

Figure 8. Pedersen and Hall conductivities resulting from 30 to $240 \mathrm{keV}$ proton precipitation centered on 17 April, 1200 UT. The three columns are the results at $180 \mathrm{~km}$, $120 \mathrm{~km}$, and $110 \mathrm{~km}$ altitude, respectively. The first two rows present the 1-D and 3-D results of the Pedersen conductivities, respectively, while the third and fourth rows are for the Hall conductivities. The conductivities are displayed on a logarithmic scale with contours every decade. The bottom row gives the relative errors of the 1-D results, which are the same for the Pedersen and Hall conductivities. 
(a) $\sigma_{\mathrm{P}}$ at $150^{\circ}$ longitude, $\log _{10}\left(\mathrm{~S} \mathrm{~m}^{-1}\right)$

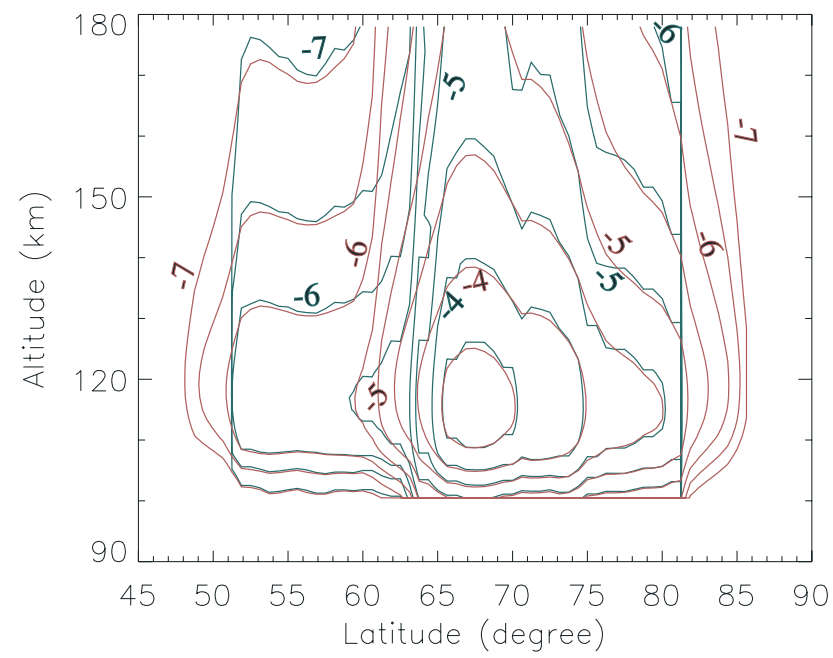

(b) Comparison of $\sigma_{P}$ at $150^{\circ}$ longitude

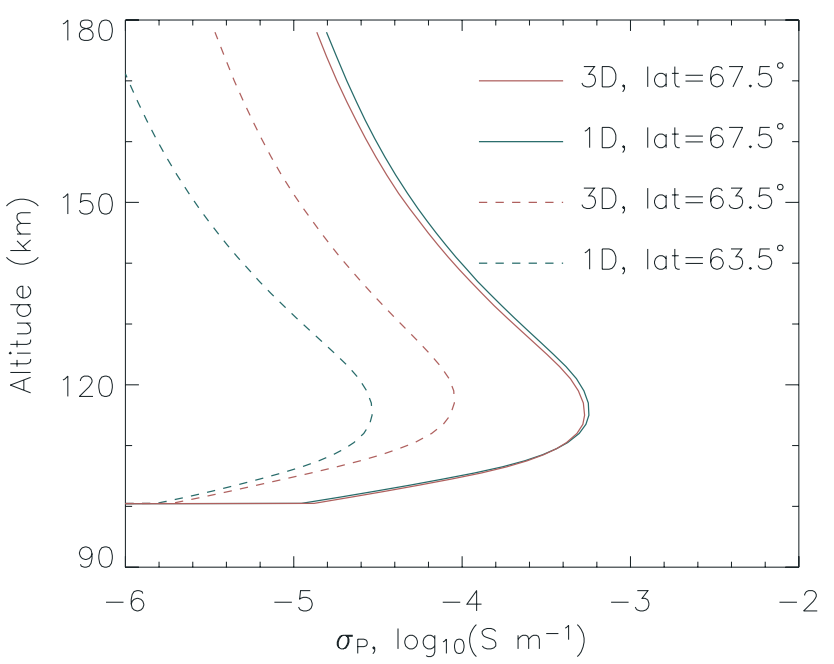

(c) $\sigma_{\mathrm{H}}$ at $150^{\circ}$ longitude, $\log _{10}\left(\mathrm{~S} \mathrm{~m}^{-1}\right)$

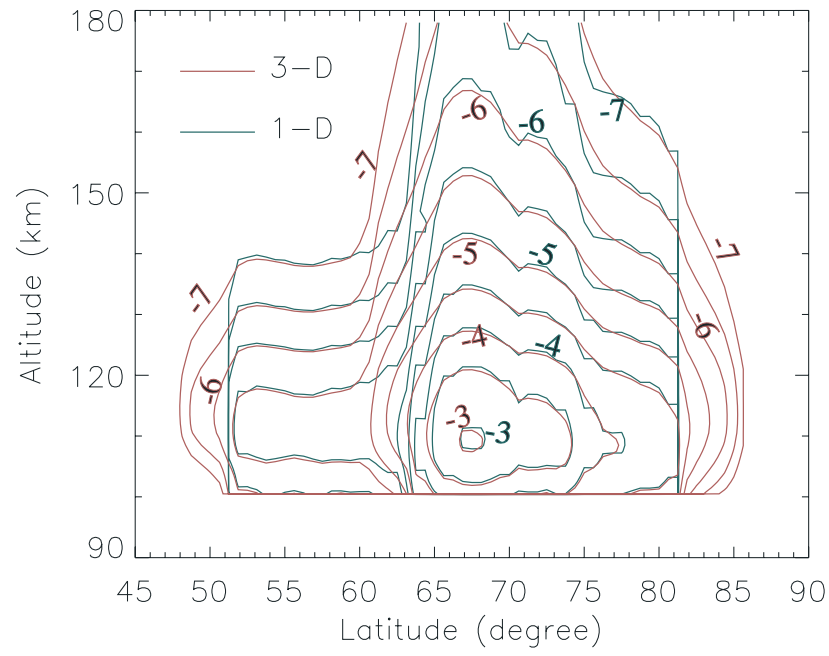

(d) Comparison of $\sigma_{H}$ at $150^{\circ}$ longitude

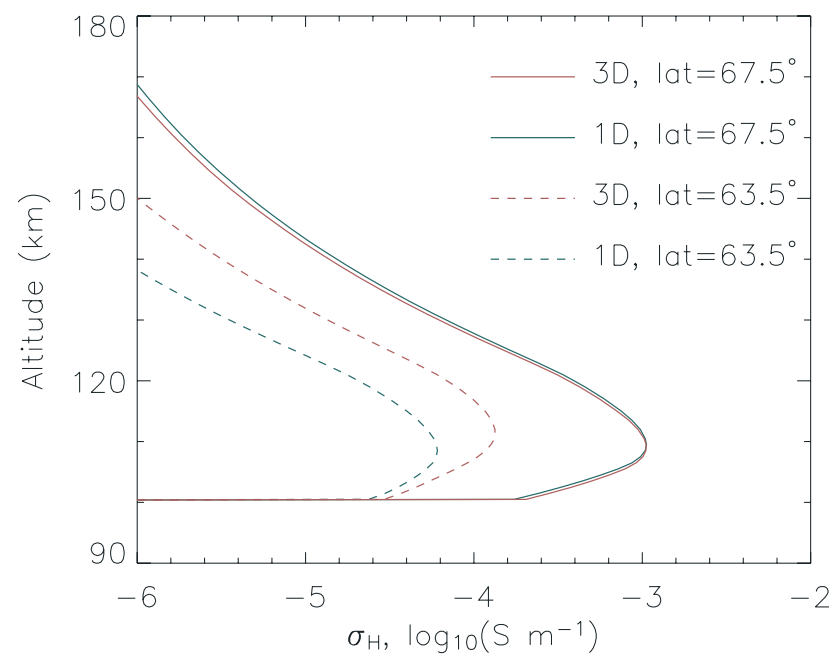

Figure 9. Comparison of the 1-D and 3-D Pedersen and Hall conductivity results from proton precipitation on 17 April, 1200 UT. Shown here is (a) a latitude-altitude profile of $\sigma_{P}$ at $150^{\circ}$ longitude, (b) an altitude profile of $\sigma_{P}$ at $150^{\circ}$ longitude and two specific latitudes $\left(67.5^{\circ}\right.$ and $\left.63.5^{\circ}\right),\left(\right.$ c) $\sigma_{H}$ at $150^{\circ}$ longitude, and (d) $\sigma_{H}$ at $150^{\circ}$ longitude and $67.5^{\circ}$ and $63.5^{\circ}$ latitudes. The conductivities in Figures $9 \mathrm{a}$ and $9 \mathrm{~b}$ are displayed on a logarithmic scale with contours every 0.5 decade. The calculation results are compared with the beam spreading effect considered (red curves) or neglected (black curves).

section 4.1 , the equatorward edge of the main proton precipitation zone (around $60^{\circ}-65^{\circ}$ latitude in the dusk sector in this example) is the region of particular interest for this study.

[37] It is worth noting that the relative errors of the 1-D results are the same for the Pedersen and for the Hall conductivities. This is because we use a branching ratio to calculate the number density of an ion species $\left(n_{i}\right)$ based on the electron concentration $\left(n_{e}\right)$. As a result, the beam spreading effect comes into play on the conductivities through the influence on the electron density (see equations (3) and (4)). In other words, the 1-D/3-D ratios of the Pedersen and Hall conductivities are the same, and both of them are equal to those of the electron density. Moreover, as already demonstrated in the ionization rate comparison in Figures 3-5, the beam spreading effect clearly functions differently at different altitude regions. As altitude decreases, the overestimation of the 1-D Pedersen and Hall conductivities declines at the particle precipitation center. The underestimation of the 1-D results at the edge is lowered as well, but is still significant.

[38] Figure 9 provides a quantitative comparison at $150^{\circ}$ geographic longitude for the 1-D and 3-D results of the Pedersen and Hall conductivities. As we have already seen in Figure 8, the Pedersen conductivities are significant in a broader altitude region than the Hall conductivities. The peak altitude of $\sigma_{H}$ is approximately $5 \mathrm{~km}$ lower than that of $\sigma_{P}$. At the center of the major proton impact zone $\left(67.5^{\circ}\right.$ latitude), the 1-D results of $\sigma_{P}$ and $\sigma_{H}$ are close to the 3-D values. There is a $\sim 7 \%$ overestimation for the $1-\mathrm{D}$ results at $120 \mathrm{~km}$ altitude. The 1-D/3-D difference almost disappears at lower altitudes. On the contrary, the beam spreading effect is more evidently demonstrated at the proton precipitation edge. At $63.5^{\circ}$ latitude, the underestimation (for both $\sigma_{P}$ and $\sigma_{H}$ ) due to the neglect of 3-D particle transport is 


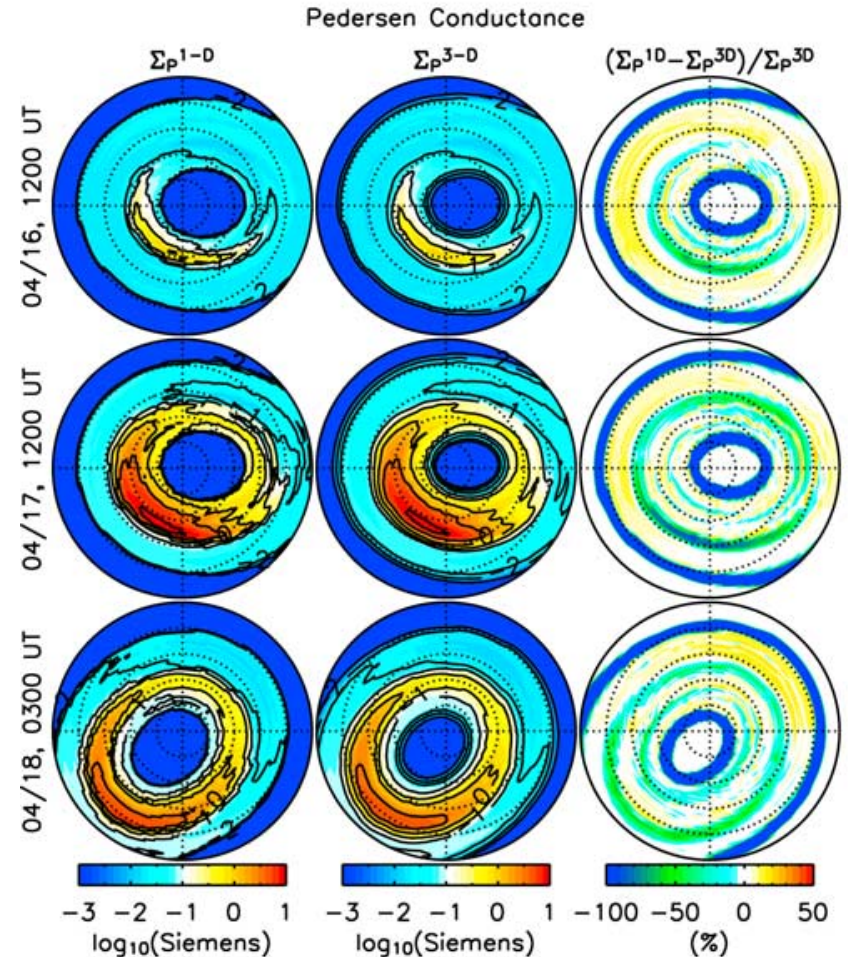

Figure 10. Pedersen conductances resulting from 30 to $240 \mathrm{keV}$ proton precipitation centered on (top row) 16 April, 1200 UT, (middle row) 17 April, 1200 UT, and (bottom row) 18 April, 0300 UT. The three columns from left to right are for the 1-D results, 3-D results, and the relative errors of the 1$D$ results, respectively. The conductances are displayed on a logarithmic scale with contours every 0.5 decade.

worse than $-70 \%$ above $120 \mathrm{~km}$ altitude. This error is around $-55 \%$ at $110 \mathrm{~km}$ altitude. Note that these errors for the 1-D results of the Pedersen and Hall conductivities are understandable, according to what we have analyzed in section 4.1 for the ionization rates. This is because conductivities are directly proportional to the square root of ionization rates (see equations (1), (3), and (4)).

\subsection{Conductance}

[39] The altitude profiles of the conductivities are height integrated to yield the Pedersen $\left(\Sigma_{P}\right)$ and Hall $\left(\Sigma_{H}\right)$ conductances, as shown in Figures 10 and 11, respectively. In accordance with the change of the proton generated ionization rates, the conductances demonstrate similar variation during the storm. That is, a strong enhancement (more than one order of magnitude) in $\Sigma_{P}$ and $\Sigma_{H}$ is generated in response to the impact of a sheath region preceding the ICME on 17 April at 1200 UT. Most of the conductance elevation is focused on the dusk sector. During the magnetic cloud driven storm (18 April, $0300 \mathrm{UT}$ ), the strength of the conductances subsides a little but covers a broader range of local time.

[40] The 1-D/3-D differences are assessed by calculating the relative errors of the 1-D conductance results. They are explicitly illustrated in the right columns of Figures 10 and 11 to facilitate comparison. Likewise, yellow-red shading and green-blue shading show the regions in which the results are overestimated and underestimated, respectively, by ignoring horizontal spatial spreading. More quantitative comparisons will be carried out later. Through the comparison of the calculation results with the beam spreading effect turned off (1-D) or on (3-D), two important conclusions can be drawn.

[41] First, it is critical to include 3-D particle scattering for a global conductance modeling. Otherwise, there is an overestimation (up to $\sim 10 \%$ ) at the center of the major proton precipitation region. More importantly, at the edge (particularly at the equatorward edge) of the major particle impact domain, a severe underestimation (around $-50 \%$ or worse) can be generated by the neglect of the beam spreading effect. Note that both the overestimation and underestimation are physically meaningful, since they occur in such a region that the conductances have a significant magnitude. It should be pointed out that the relative errors of the 1-D results in the sheath driven storm (17 April, 1200 UT) are considerably larger than those in the magnetic cloud driven storm (18 April, 0300 UT). This can be explained by the steeper gradient in the energy flux distribution as seen in Figure 1.

[42] Second, the lateral spreading of a proton arc has a larger effect on the Pedersen conductances than on the Hall conductances. This difference is reflective of the altitude dependence of the beam spreading effect. As we discussed previously, the beam spreading effect plays a more significant role at high altitudes than at low altitudes. Moreover, as already demonstrated in Figures 8 and 9, the peak altitude of the Pedersen conductivities is around $5 \mathrm{~km}$ higher than that of the Hall conductivities. As a result, it is expected that the induced relative errors of the 1-D results are more

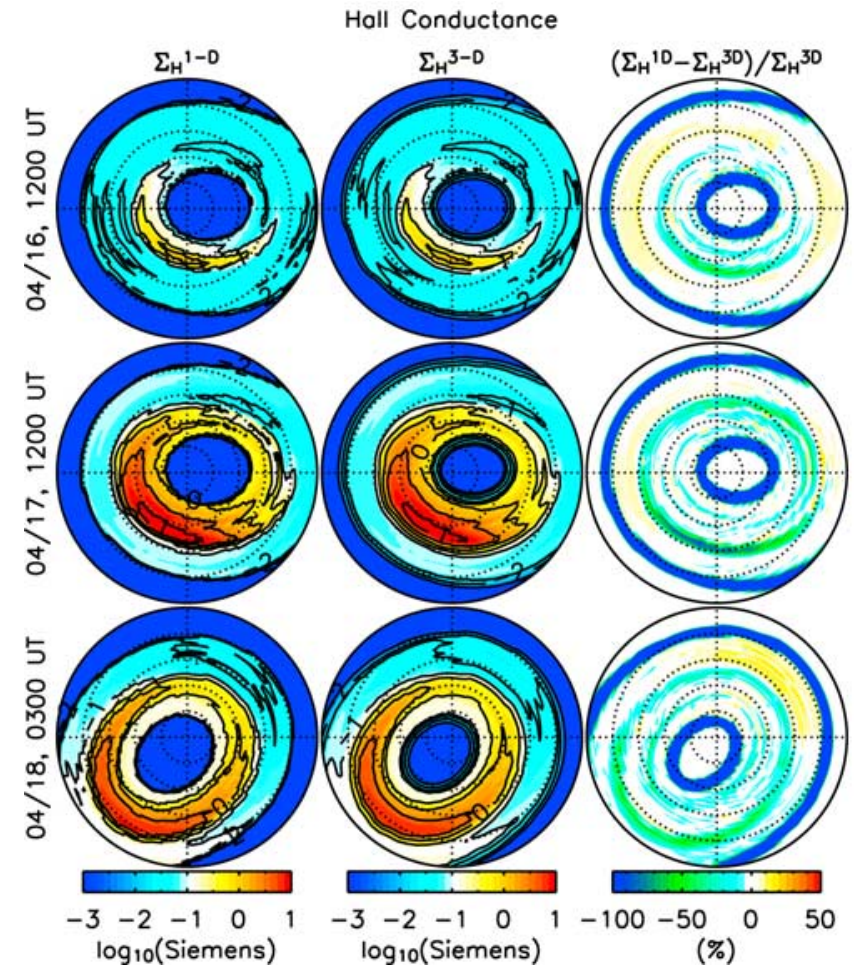

Figure 11. Similar to Figure 10 but for the Hall conductances. 
Table 1. One-Dimensional/Three-Dimensional Comparison for the Pedersen and Hall Conductances at Two Specific Geographic Locations on 17 April, 1200 UT

\begin{tabular}{lccc}
\hline & $\Sigma^{3 D}, \mathrm{~S}$ & $\Sigma^{1 D}, \mathrm{~S}$ & $\left(\Sigma^{1 D}-\Sigma^{3 D}\right) / \Sigma^{3 D}, \%$ \\
\hline \multirow{4}{*}{ Pedersen } & $150^{\circ}$ longitude, $67.5^{\circ}$ latitude \\
Hall & 12.35 & 13.20 & 6.88 \\
& 15.95 & 16.10 & 0.94 \\
Pedersen & $150^{\circ}$ longitude, $63.5^{\circ}$ latitude \\
Hall & 2.21 & 0.69 & -68.78 \\
\hline
\end{tabular}

significant for $\Sigma_{P}$ than for $\Sigma_{H}$. Given that Joule heating is proportional to $\Sigma_{P}$ rather than $\Sigma_{H}$, the beam spreading effect is important for the Joule heating calculation.

[43] Table 1 presents the $1-\mathrm{D} / 3-\mathrm{D}$ comparison for the Pedersen and Hall conductances on 17 April at 1200 UT. As an example, the comparison is made at two specific locations: one at the center of proton precipitation domain $\left(150^{\circ}\right.$ longitude, $67.5^{\circ}$ latitude), the other at the equatorward edge $\left(150^{\circ}\right.$ longitude, $63.5^{\circ}$ latitude). The conclusions we have drawn above from Figures 10 and 11 are quantitatively supported in Table 1.

[44] To better understand the influence of the beam spreading effect, it is more appropriate to compare the 1-D and 3-D conductances in a geomagnetic coordinate system. Figure 12 presents the Pedersen and Hall conductances with respect to magnetic latitude at a given magnetic local time (MLT) (across the major precipitation domain in the dusk sector). The open circles mark the equatorward edge of the 3-D conductance distributions, whose magnitudes exceed a background value. The threshold value is set to be $0.5 \mathrm{~S}$ for Pedersen conductance and $1.0 \mathrm{~S}$ for Hall conductance. The open squares mark the upper limits in magnetic latitude (still equatorward of the peak conductances), where the 1-D/3-D ratios are equal to $90 \%$.

[45] The latitude regions bounded by the open circle and open square (with the same color) represent a significance domain, in which significant conductances (larger than background values) would be seriously underestimated by the neglect of the beam spreading effect. That is, the relative errors of the 1-D results in this domain are at least worse than $-10 \%$ (down to around $-90 \%$ in this example). By taking into account horizontal spreading in the particle transport, the equatorward edges extend down to $\sim 55^{\circ}$ CML for Pedersen conductance and $\sim 56^{\circ} \mathrm{CML}$ for Hall conductance. This equatorward extension of the high conductance region by several degrees in magnetic latitude highlights the importance of the beam spreading effect, as the development of storm-time phenomena can be impacted. Further discussion will be made in the next section.

[46] In addition, as we have seen in Figures 10 and 11, there is a general overestimation (up to $10 \%$ ) for the 1-D results of the Pedersen and Hall conductances at the center of the major particle impact region. The 1-D/3-D difference at the poleward edge is almost negligible.

[47] Let us extend this analysis of the lower and upper limits of the significance domain (as defined above in Figure 12). Figure 13 shows this significance domain in a magnetic local time span from dusk to postmidnight during the sheath driven storm (17 April, 1200 UT). It is seen that in a wide MLT range (from $\sim 20$ hour in the premidnight sector to $\sim 1$ hour in the postmidnight sector), the beam spreading effect is important for an accurate understanding of the global proton generated conductance distribution. There is a considerable significance domain associated with the beam spreading effect equatorward of the major proton precipitation region. The peak width of the significance domain is $>3.5^{\circ}$ in CML for the Pedersen conductances, and $>2.5^{\circ}$ for the Hall conductances. At 22.8 hour MLT, the significance domain is as wide as $4.4^{\circ}$ and $3.5^{\circ}$ in CML for the Pedersen and Hall conductances, respectively. As a comparison, in the magnetic cloud driven storm (18 April, $0300 \mathrm{UT})$, the width drops to $>2.5^{\circ}$ and $>1.5^{\circ}$ in $\mathrm{CML}$ for $\Sigma_{P}$ and $\Sigma_{H}$, respectively (not shown here). However, the local time extent is larger, and covers the whole dusk sector and a considerable portion of the post-midnight sector.

\section{Discussion}

[48] We have applied our 3-D Monte Carlo ion transport model [Fang et al., 2004] to calculate the Pedersen and Hall conductances resulting from the proton precipitation during the 17-18 April 2002 magnetic storms. By comparing the 1-D/3-D results (with the beam spreading effect switched off or on), it is well illustrated that it is critical to include the beam spreading effect in a global model of the conductance distribution. In the current study, however, our effort is concentrated on the high energy proton input; that is, 30$240 \mathrm{keV}$ ion measurements by the NOAA/POES satellites

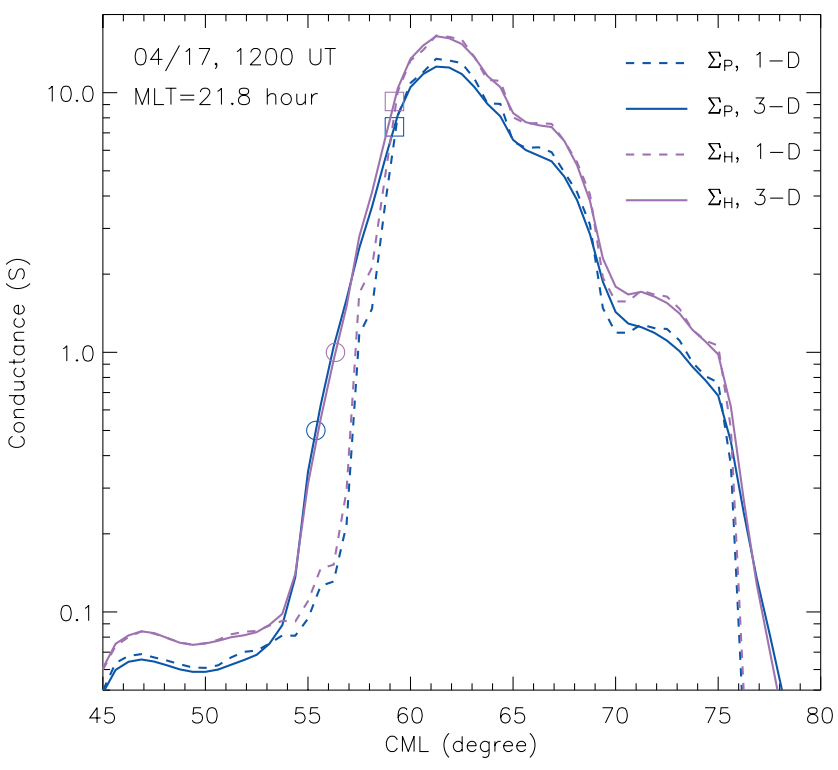

Figure 12. Magnetic latitude profiles of the Pedersen and Hall conductances at 21.8 hour magnetic local time, resulting from the $30-240 \mathrm{keV}$ proton precipitation centered on 17 April, 1200 UT. The results are shown by dashed and solid curves when the beam spreading effect is neglected or considered, respectively. The open circles mark the locations at the equatorward edge of the 3-D conductances, where $\Sigma_{P}^{3 D}=0.5 \mathrm{~S}$ (blue circle) and $\Sigma_{H}^{3 D}=1 \mathrm{~S}$ (red circle), respectively. The open squares indicate the locations equatorward of the peak conductances, where the 1-D/3-D ratio is $90 \%$. 


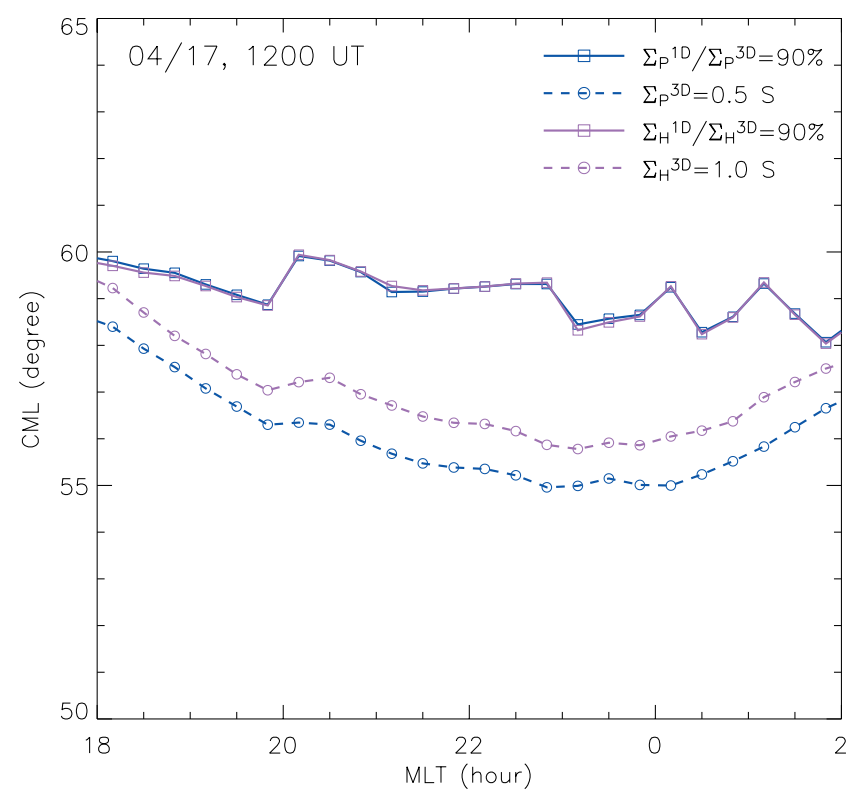

Figure 13. Lower and upper magnetic latitude limits of a significance domain for the beam spreading effect in the conductance calculation of the proton precipitation centered on 17 April, 1200 UT. The plots are shown versus magnetic local time from dusk to postmidnight. The lower limits (dashed curves with open circles) represent the equatorward edges at which the 3-D conductances approach to background values $\left(0.5 \mathrm{~S}\right.$ for $\Sigma_{P}^{3 D}$, and $1.0 \mathrm{~S}$ for $\left.\Sigma_{H}^{3 D}\right)$. The upper limits (solid curves with open squares) mark the locations where the 1-D/3-D ratios $\left(\Sigma^{1 D} / \Sigma^{3 D}\right)$ are $90 \%$.

(see paper 1). This energy band represents the bulk of the ring current and a high energy tail of the plasma sheet ion precipitation.

[49] It is of interest in future studies to include the lowenergy $(<30 \mathrm{keV})$ protons in the calculation. Note that the error of the 1-D approximation generally becomes more serious for lower-energy proton precipitation, as demonstrated by Fang et al. [2004, 2005]. It is therefore expected that the beam spreading plays an even more important role in the global conductance calculation when $<30 \mathrm{keV}$ protons are included.

[50] In the present version of the ionization calculation, secondary electrons are omitted from consideration. The further ionization production by these secondary electrons can be approximately estimated by multiplying the primary ionization rate by $0.006 E_{0}$ [Lilensten and Galand, 1998], where $E_{0}$ is the characteristic energy of a Maxwellian proton flux in $\mathrm{keV}$. A significance domain equatorward of the major proton precipitation region is defined in section 4.4 in association with the beam spreading effect. It is representative of a region in the CML-MLT coordinate system, in which the Pedersen and Hall conductances would be seriously underestimated with the neglect of 3-D particle scattering. The lower limits in magnetic latitude correspond to the locations where the conductances drop below preset threshold values $\left(0.5 \mathrm{~S}\right.$ for $\Sigma_{P}, 1.0 \mathrm{~S}$ for $\left.\Sigma_{H}\right)$. The upper limits mark the poleward edge of the significance domain, where the $1-\mathrm{D} / 3-\mathrm{D}$ conductance ratio is $90 \%$. The inclusion of the additional ionization due to secondary electrons will enhance the resulting conductances, making the lower limits of the high conductance region move equatorward. Given the fact that the beam spreading effect is a unique feature of ion transport, the consideration of secondary electrons will have little effect on the upper latitudinal limits. As a consequence, it is expected that after considering the ionization by secondary electrons, the significance domain is enlarged; that is, it is of more importance to include 3-D particle transport in the global conductance calculation.

[51] As seen in Figures 10 and 11, the beam spreading effect is also significant in the conductance distribution even during the pre-storm conditions (16 April, 1200 UT). Note that in accordance with the study of Coumans et al. [2004], the relative contribution of precipitating protons in the Pedersen and Hall conductances is globally more important during quiet periods than during active periods. At low $K p$ values, the increase in conductances due to additional protons is globally more than $50 \%$ in comparison with the conductances generated by electrons only. In contrast, this number decreases to less than $6 \%$ at high $K p$ values. The beam spreading analyses performed in the current study suggest that 3-D particle transport should be also stressed during quiet time, as the role of precipitating protons in the ionospheric electrodynamics rises.

[52] The current study is for the 17-18 April 2002 geomagnetic storms, which are actually moderate in their peak activity level. The findings in paper 1 report that the midnight equatorward boundary of precipitating $30-$ $240 \mathrm{keV}$ proton number fluxes during these events approaches down to around $55.5^{\circ}$ in CML. However, new satellite observations during major magnetic storms indicate that strong ion/neutral particle precipitation can extend to magnetic latitudes much more equatorward (below $33^{\circ}$ ) [e.g., Kozyra et al., 2004; Zhang et al., 2006]. Spreading of the incident beam from its original magnetic field line brings the ionization and conductances to even lower latitudes. The conductance change at low latitudes has a large effect on the penetration of stormtime convection electric fields to the equator [e.g., Spiro et al., 1988; Fejer et al., 1990; Ridley and Liemohn, 2002]. These electric fields are responsible for triggering equatorial irregularities and large-scale redistribution of ionospheric plasma [c.f., Burke et al., 2000].

[53] Subauroral polarization streams (SAPS) is an interesting phenomenon in the midlatitude ionosphere related to the conductance distribution. SAPS refers to the poleward directed electric fields at subauroral latitudes that drive strong sunward flows on the duskside and nightside [Foster and Burke, 2002; Foster and Vo, 2002]. The development of SAPS due to closure of the partial ring current through the ionosphere critically depends on the conductance gradients equatorward of the auroral oval, where electron precipitation gives way to ion precipitation [e.g., Ridley and Liemohn, 2002; Fok et al., 2003; Garner et al., 2004]. Numerical simulations using the Rice Convection Model (RCM) [Wolf, 1983] successfully reproduce SAPS, however, with the latitudinal locations poleward of where the data indicate (up to $\sim 5^{\circ}$ discrepancy) [e.g., Garner et al., 2004; Sazykin et al., 2005]. Three factors are of particular interest in this problem. First, SAPs is associated with low conductances equatorward of the auroral oval. Second, auroral electron precipitation is the only particle contribu- 
tion to the conductance distribution employed in RCM. Third, ion precipitation occurs equatorward of electron precipitation in the evening sector [Hardy et al., 1989]. Therefore one possible resolution to the SAPS latitudinal location problem is to include ion precipitation in the conductance model. Given that the spreading of an incident ion beam can extend the high conductance domain equatorward (up to $4.4^{\circ}$ in magnetic latitude), it is expected that predicted SAPS locations in RCM will be in a better agreement with observations after taking into account ion precipitation as well as its beam spreading.

[54] Recently, many efforts have been made to investigate the influence of conductance on the development of the storm-time ring current [Khazanov et al., 2003; Ebihara et al., 2004; Ebihara and Fok, 2004; Liemohn et al., 2004, 2005]. It is shown that when different conductance models are used, important alterations occur to the electric potential pattern and thus the ring current. It is worth noting that precipitating protons are usually neglected in the conductance models employed in these studies except that of Khazanov et al. [2003]. In Khazanov et al.'s model, the conductance due to proton precipitation is assessed using Galand and Richmond's [2001] simplified formulas derived from 1-D modeling. That is, the beam spreading effect is not included. However, our calculation results in this paper imply that a considerable domain (up to $4.4^{\circ}$ in magnetic latitude) exists equatorward of proton precipitation region, where the conductances could be seriously underestimated under the 1-D particle transport approximation. The resulting influence on the ring current development deserves special attention. It is beyond the scope of this study but should be the topic of a future study.

\section{Summary and Conclusion}

[55] This paper presents the global Pedersen and Hall conductance distribution generated by energetic proton precipitation during the 17-18 April 2002 geomagnetic storms and provides the first assessment of the influence of the beam spreading effect. The horizontal beam spread is a unique feature of ion precipitation, which is resulting from charge exchange and electron stripping collisions within an incident ion beam [Davidson, 1965; Johnstone, 1972; Igelesias and Vondrak, 1974; Kozelov, 1993; Synnes et al., 1998; Lorentzen, 2000; Fang et al., 2004, 2005]. This effect can only be accurately captured in a model capable of 3-D particle transport calculation. A recently developed 3-D Monte Carlo ion transport model [Fang et al., 2004] is a major research tool and exactly fits the needs for this study.

[56] In this paper the topside ion energy input during the selected storms is characterized by the NOAA/POES global $30-240 \mathrm{keV}$ proton precipitation patterns constructed at a 3 -hour cadence in paper 1 . The resulting ionization rates are calculated using our 3-D Monte Carlo ion transport model [Fang et al., 2004]. The Pedersen and Hall conductances and thus their global distribution are derived. In the sheath driven storm, a rapid and strong enhancement (more than one order of magnitude) in the conductances is generated in response to the impact of a shocked solar wind preceding the ICME on 17 April at 1200 UT. Most of the conductance elevation is focused on the dusk sector, with the peak values exceeding $10 \mathrm{~S}$. In the selected magnetic cloud driven storm (18 April, 0300UT), the strength of the conductances subsides a little (more than $5 \mathrm{~S}$ at the peak region) but covers a broader range of local time.

[57] The effect of 3-D particle scattering on the conductance distribution is quantitatively analyzed by comparing the calculation results with the beam spreading effect turned off (1-D results) or on (3-D results). Our findings stress that it is critical for a conductance model to include the beam spreading effect in the proton transport calculation. With the neglect of 3-D particle scattering, a moderate overestimation (up to $\sim 10 \%$ ) occurs at the center of the major ion precipitation region. More importantly, a severe underestimation (around $-50 \%$ or worse) can be induced at the precipitation edge (particularly at the equatorward edge). Moreover, after the consideration of 3-D particle scattering in the calculation, the equatorward edge of the high conductance region can extend down to around $55^{\circ}$ magnetic latitude in the storm events studied in this paper.

[58] It is found that the beam spreading effect of proton precipitation has a larger influence on the Pedersen conductances than on the Hall conductances. This is in accordance with the altitude dependence of both conductivity profiles and the spreading. For a given energetic proton injection, the resulting Pedersen conductivities peak at altitudes $\sim 5 \mathrm{~km}$ higher than those for the Hall conductivities. Moreover, the beam spreading effect is also altitude dependent. The effect of beam spreading of proton precipitation becomes less important as altitude decreases [Fang et al., 2005]. As a consequence, the beam spreading effect needs more attention in the Pedersen conductance calculation.

[59] In this paper a significance domain is defined in association with the beam spreading effect. It represents a domain equatorward of the major proton precipitation region in the evening sector, in which significant Pedersen and Hall conductances would be seriously underestimated with the neglect of the beam spreading effect. The lower magnetic latitude boundary corresponds to the locations at which the conductances drop below preset background values $(0.5 \mathrm{~S}$ and $1.0 \mathrm{~S}$ for the Pedersen and Hall conductances, respectively). The upper boundary marks the poleward edge of the significance domain, at which the $1-\mathrm{D} / 3-\mathrm{D}$ conductance ratio is $90 \%$. The significance domain in the selected sheath driven storm (17 April, 1200 UT) is as wide as $>3.5^{\circ}$ in CML for the Pedersen conductances and $>2.5^{\circ}$ for the Hall conductances across most of the evening sector. At some magnetic local times, the width becomes $4.4^{\circ}$ and $3.5^{\circ}$ in CML for the Pedersen and Hall conductances, respectively. In the selected magnetic cloud driven storm (18 April, $0300 \mathrm{UT}$ ), however, the width drops to $>2.5^{\circ}$ and $>1.5^{\circ}$ in CML for $\Sigma_{P}$ and $\Sigma_{H}$, respectively. The local time extent is enlarged and covers the whole dusk sector and a considerable portion of the postmidnight sector.

[60] The additional conductivity produced by proton precipitation and its resulting lateral spread could have large impacts on the development of storm-time phenomena. In particular, it could influence the electromagnetic coupling of the ionosphere and magnetosphere, especially in the evening sector at midlatitudes. Processes such as subauroral polarization streams, ionospheric storm enhanced density structures, ring current development, and plasmaspheric 
erosion could all be affected by the increased conductance from proton precipitation.

[61] Acknowledgments. We would like to thank Aaron Ridley for enriching discussions on the topic of this study. This work was supported by NASA grants NAG 5-5030 and NAG 5-11831 and NSF grant ATM0090165.

[62] Zuyin Pu thanks Wenbin Wang and Anthony Lui for their assistance in evaluating this paper.

\section{References}

Aksnes, A., J. Stadsnes, J. Bjordal, N. Tgaard, R. R. Vondrak, D. L. Detrick, T. J. Rosenberg, G. A. Germany, and D. Chenette (2002), Instantaneous ionospheric global conductance maps during an isolated substorm, Ann. Geophys., 20, 1181

Basu, B., J. R. Jasperse, R. M. Robinson, R. R. Vondrak, and D. S. Evans (1987), Linear transport theory of auroral proton precipitation: A comparison with observations, J. Geophys. Res., 92, 5920.

Bilitza, D., (Ed.) (1990), International Reference Ionosphere 1990, NSSDC 90-22, Natl. Space Sci. Data Cent., Greenbelt, Md.

Brekke, A., and J. Moen (1993), Observations of high latitude ionospheric conductances, J. Atmos. Terr. Phys., 55, 1493.

Burke, W. J., A. G. Rubin, N. C. Maynard, L. C. Gentile, P. J. Sultan, F. J. Rich, O. de La Beaujardiere, C. Y. Huang, and G. R. Wilson (2000), Ionospheric disturbances observed by DMSP at middle to low latitudes during the magnetic storm of June 4-6, 1991, J. Geophys. Res., 105, 18,391 .

Clauer, C. R., X. Cai, D. Welling, A. DeJong, and M. G. Henderson (2006), Characterizing the 18 April 2002 storm-time sawtooth events using ground magnetic data, J. Geophys. Res., 111, A04S90, doi:10.1029/ 2005JA011099.

Coumans, V., J.-C. Gérard, B. Hubert, M. Meurant, and S. B. Mende (2004), Global auroral conductance distribution due to electron and proton precipitation from IMAGE-FUV observations, Ann. Geophys., 22 1595.

Davidson, G. T. (1965), Expected spatial distribution of low energy protons precipitated in the auroral zones, J. Geophys. Res., 70, 1061.

Ebihara, Y., and M.-C. Fok (2004), Posstmidnight storm-time enhancement of tens-of-keV proton flux, J. Geophys. Res., 109, A12209, doi:10.1029/ 2004JA010523.

Ebihara, Y., M.-C. Fok, R. A. Wolf, T. J. Immel, and T. E. Moore (2004), Influence of ionospheric conductivity on the ring current, $J$. Geophys. Res., 109, A08205, doi:10.1029/2003JA010351.

Fang, X., M. W. Liemohn, J. U. Kozyra, and S. C. Solomon (2004), Quantification of the spreading effect of auroral proton precipitation, J. Geophys. Res., 109, A04309, doi:10.1029/2003JA010119.

Fang, X., M. W. Liemohn, J. U. Kozyra, and S. C. Solomon (2005), Study of the proton arc spreading effect on primary ionization rates, J. Geophys. Res., 110, A07302, doi:10.1029/2004JA010915.

Fang, X., M. W. Liemohn, J. U. Kozyra, D. S. Evans, A. D. DeJong, and B. A. Emery (2007), Global $30-240 \mathrm{keV}$ proton precipitation in the 17-18 April 2002 geomagnetic storms: 1. Patterns, J. Geophys. Res., doi:10.1029/2006JA011867, in press.

Fedder, J., and J. Lyon (1987), The solar wind-magnetosphere-ionosphere current-voltage relationship, Geophys. Res. Lett., 14, 880.

Fejer, B. G., R. W. Spiro, R. A. Wolf, and J. C. Foster (1990), Latitudinal variation of perturbation electric fields during magnetically disturbed periods: 1988 SUNDIAL observations and model results, Ann. Geophys., $8,441$.

Fok, M. -C., et al. (2003), Global ENA image simulations, Space Sci. Rev., 109, 77.

Foster, J. C., and W. J. Burke (2002), Saps: A new characterization for subauroral electric fields, Eos Trans. AGU, 83, 393.

Foster, J. C., and H. B. Vo (2002), Average characteristics and activity dependence of the subauroral polarization stream, J. Geophys. Res., 107(A12), 1475, doi:10.1029/2002JA009409.

Fuller-Rowell, T. J., and D. S. Evans (1987), Height-integrated Pederson and Hall conductivity patterns inferred from the TIROS-NOAA satellite data, J. Geophys. Res., 92, 7606.

Galand, M., and A. D. Richmond (2001), Ionospheric electrical conductances produced by auroral proton precipitation, J. Geophys. Res., 106, 117.

Galand, M., J. Lilensten, W. Kofman, and R. B. Sidge (1997), Proton transport model in the ionosphere: 1. Multistream approach of the transport equations, J. Geophys. Res., 102, 22,261.

Galand, M., R. G. Roble, and D. Lummerzheim (1999), Ionization by energetic protons in Thermosphere-Ionosphere Electrodynamics General Circulation Model, J. Geophys. Res., 104, 27,973.
Galand, M., T. J. Fuller-Rowell, and M. V. Codrescu (2001), Response of the upper atmosphere to auroral protons, J. Geophys. Res., 106, 127.

Garner, T. W., R. A. Wolf, R. W. Spiro, W. J. Burke, B. G. Fejer, S. Sazykin, J. L. Roeder, and M. R. Hairston (2004), Magnetospheric electric fields and plasma sheet injection to low L-shells during the 4-5 June 1991 magnetic storm: Comparison between the Rice Convection Model and observations, J. Geophys. Res., 109, A02214, doi:10.1029/ 2003JA010208

Gussenhoven, M. S., D. A. Hardy, and N. Heinemann (1987), The equatorward boundary of auroral ion precipitation, J. Geophys. Res., 92, 3273.

Hardy, D. A., M. S. Gussenhoven, and E. Holeman (1985), A statistical model of auroral electron precipitation, J. Geophys. Res., 90, 4229.

Hardy, D. A., M. S. Gussenhoven, and R. Raistrick (1987), Statistical and functional representations of the pattern of auroral energy flux, number flux, and conductivity, J. Geophys. Res., 92, 12,275.

Hardy, D. A., M. S. Gussenhoven, and D. Brautigam (1989), A statistical model of auroral ion precipitation, J. Geophys. Res., 94, 370.

Hardy, D. A., W. McNeil, M. S. Gussenhoven, and D. Brautigam (1991), A statistical model of auroral ion precipitation, 2, Functional representation of the average patterns, J. Geophys. Res., 96, 5539.

Hedin, A. E. (1991), Extension of the miss thermosphere model into the middle and lower atmosphere, J. Geophys. Res., 96, 1159.

Henderson, M. G., G. D. Reeves, R. Skoug, M. T. Thomsen, M. H. Denton, S. B. Mende, T. J. Immel, P. C. Brandt, and H. J. Singer (2006), Magnetospheric and auroral activity during the 18 April 2002 sawtooth event, J. Geophys. Res., 111, A01S90, doi:10.1029/2005JA011111.

Igelesias, G. E., and R. R. Vondrak (1974), Atmospheric spreading of protons in auroral arcs, J. Geophys. Res., 79, 280.

Jasperse, J. R., and B. Basu (1982), Transport-theoretic solutions for auroral proton and $\mathrm{H}$ atom fluxes and related quantities, J. Geophys. Res., 87, 811.

Johnstone, A. D. (1972), The spreading of a proton beam by the atmosphere, Planet. Space Sci., 20, 292.

Khazanov, G. V., M. W. Liemohn, T. S. Newman, M. -C. Fok, and R. W. Spiro (2003), Self-consistent magnetosphere-ionosphere coupling: Theoretical studies, J. Geophys. Res., 108(A3), 1122, doi:10.1029/ 2002JA009624

Kozelov, B. V. (1993), Influence of the dipolar magnetic field on transport of proton-H atom fluxes in the atmosphere, Ann. Geophys., 11, 697.

Kozyra, J., et al (2004), Comparison of magnetospheric conditions during three superstorms driven by the extreme solar events in 2003, paper presented at Solar Extreme Events Symposium, Moscow State Univ., Moscow, Russia, $12-14$ July .

Langel, R. A. (1992), International Geomagnetic Reference Field: The sixth generation, J. Geomagn. Geoelectr., 44, 679.

Liemohn, M. W., A. J. Ridley, D. L. Gallagher, D. M. Ober, and J. U. Kozyra (2004), Dependence of plasmaspheric morphology on the electric field description during the recovery phase of the April 17, 2002 magnetic storm, J. Geophys. Res., 109, A03209, doi:10.1029/2003JA010304.

Liemohn, M. W., A. J. Ridley, P. C. Brandt, D. L. Gallagher, J. U. Kozyra, D. M. Ober, D. G. Mitchell, E. C. Roelof, and R. DeMajistre (2005), Parametric analysis of nightside conductance effects on innner magnetospheric dynamics for the 17 April 2002 storm, J. Geophys. Res., 110, A12S22, doi:10.1029/2005JA011109.

Lilensten, J., and M. Galand (1998), Proton-electron precipitation effects on the electron production and density above EISCAT (Tromsø) and ESR, Ann. Geophys., 16, 1299.

Lorentzen, D. A. (2000), Latitudinal and longitudinal dispersion of energetic auroral protons, Ann. Geophys., 18, 81 .

Lu, G., A. D. Richmond, J. M. Ruohoniemi, R. A. Greenwald, M. Hairston, F. J. Rich, and D. S. Evans (2001), An investigation of the influence of data and model inputs on assimilative mapping of ionospheric electrodynamics, J. Geophys. Res., 106, 417.

Lummerzheim, D., M. H. Rees, J. D. Craven, and L. A. Frank (1991), Ionospheric conductances derived from DE-1 auroral images, J. Atmos. Terr. Phys., 53, 281.

Lundblad, J. A., F. Søraas, and K. Aarsnes (1979), Substorm morphology of greater than $100 \mathrm{keV}$ protons, Planet. Space Sci., 27, 841.

Pesnell, W. D., K. Omidvar, and W. R. Hoegy (1993), Momentum transfer collision frequency of $\mathrm{O}^{+} \mathrm{O}$, Geophys. Res. Lett, 20, 1343.

Raben, V. J., D. E. Evans, H. H. Sauer, S. R. Sahm, and M. Huynh (1995), TIROS/NOAA satellite space environment monitor data archive documentation: 1995 update, in Tech. Memo. ERL SEC-86, NOAA, Boulder, Colo.

Raeder, J., R. McPherron, L. Frank, S. Kokubun, G. Lu, T. Mukai, W. Paterson, J. Sigwarth, H. Singer, and J. Slavin (2001), Global simulation of the Geospace Environment Modeling substorm challenge event, J. Geophys. Res., 106, 281.

Richmond, A. D. (1995), Ionospheric electrodynamics, in Handbook of Atmospheric Electrodynamics, vol. 2, edited by H. Volland, p. 249, CRC Press, Boca Raton, Fla. 
Richmond, A. D., and Y. Kamide (1988), Mapping electrodynamic features of the high-latitude ionosphere from localized observations: Technique, J. Geophys. Res., 93, 5741.

Ridley, A. J., and M. W. Liemohn (2002), A model-derived storm time asymmetric ring current driven electric field description, J. Geophys. Res., 107(A8), 1151, doi:10.1029/2001JA000051.

Ridley, A. J., T. I. Gombosi, and D. L. DeZeeuw (2004), Ionospheric control of the magnetosphere: Conductance, Ann. Geophys., 22, 567.

Sazykin, S., R. W. Spiro, R. A. Wolf, F. R. Toffoletto, N. Tsyganenko, J. Goldstein and M. Hairston (2005), Modeling inner magnetospheric electric fields: Latest self-consistent results, in The Inner Magnetosphere: Physics and Modeling, Geophys. Monogr. Ser., vol. 155 , edited by T. I. Pulkkinen, N. A. Tsyganenko, and R. H. W. Friedel, p. 263, AGU, Washington, D. C.

Senior, C., J. R. Sharber, O. De La Beaujardière, R. A. Heelis, D. S. Evans, J. D. Winningham, M. Sugiura, and W. R. Hoegy (1987), $E$ and $F$ region study of the evening sector auroral oval: A Chatanika/Dynamics Explorer 2/NOAA 6 comparison, J. Geophys. Res., 92, 2477.

Sharber, J. R. (1981), The continuous (diffuse) aurora and auroral-E ionization, in Physics of Space Plasma, vol. 7, edited by T. S. Chang, B. Coppi, and J. R. Jasperse, p. 115, Scientific, Gainesville, Fla.

Søraas, F., H. R. Lindalen, K. Mâseide, A. Egeland, T. A. Sten, and D. S. Evans (1974), Proton precipitation and the $H_{\beta}$ emission in a postbreakup auroral glow, J. Geophys. Res., 79, 1851.

Spiro, R. W., P. H. Reiff, and L. J. Maher Jr. (1982), Precipitating electron energy flux and auroral zone conductances: An empirical model, J. Geophys. Res., 87, 8215.

Spiro, R. W., R. A. Wolf, and B. G. Fejer (1988), Penetration of highlatitude-electric-field effects to low latitudes during SUNDIAL 1984 Ann. Geophys., 6, 39.
Strickland, D. J., R. E. Daniell Jr., J. R. Jasperse, and B. Basu (1993), Transport-theoretic model for the electron-proton-hydrogen atom aurora: 2. Model results, J. Geophys. Res., 98, 21,533

Synnes, S. A., F. Søraas, and J. P. Hansen (1998), Monte-Carlo simulations of proton aurora, J. Atmos. Sol. Terr. Phys., 60, 1695.

Urban, A. (1981), Measurements of low energy auroral ions, Planet. Space Sci., 29, 1353.

Vickrey, J. F., R. R. Vondrak, and S. J. Matthews (1982), Energy deposition by precipitating particles and Joule dissipation in the auroral ionosphere, J. Geophys. Res., 87, 5184

Wallis, D. D., and E. E. Budzinski (1981), Empirical models of height integrated conductivities, J. Geophys. Res., 86, 125.

Wolf, R. A. (1983), The quasi-static (slow-flow) region of the magnetosphere, in Solar-Terrestrial Physics, edited by R. L. Carovillano and J. M. Forbes, 303, Springer, New York.

Zhang, Y., L. J. Paxton, J. U. Kozyra, H. Kil, and P. C. Brandt (2006), Nightside thermospheric FUV emissions due to energetic neutral atom precipitation during magnetic superstorms, J. Geophys. Res., 111, A09307, doi:10.1029/2005JA011152.

X. Fang, M. W. Liemohn, and J. U. Kozyra, Space Physics Research Laboratory, University of Michigan, 2455 Hayward Street, Ann Arbor, M 48109-2143, USA. (xhfang@umich.edu; liemohn@umich.edu; jukozyra@ umich.edu)

D. S. Evans, Space Environment Center, National Oceanic and Atmospheric Administration, 325 Broadway, Boulder, CO 80303, USA. (david.s.evans@noaa.gov) 